Document downloaded from:

http://hdl.handle.net/10251/161701

This paper must be cited as:

Jornet, M.; Calatayud, J.; Le Maître, OP.; Cortés, J. (2020). Variance reduction methods and multilevel Monte Carlo strategy for the density estimation of random second order linear differential equations solutions. International Journal for Uncertainty Quantification. 10(5):467-497. https://doi.org/10.1615/Int.J.UncertaintyQuantification.2020032659

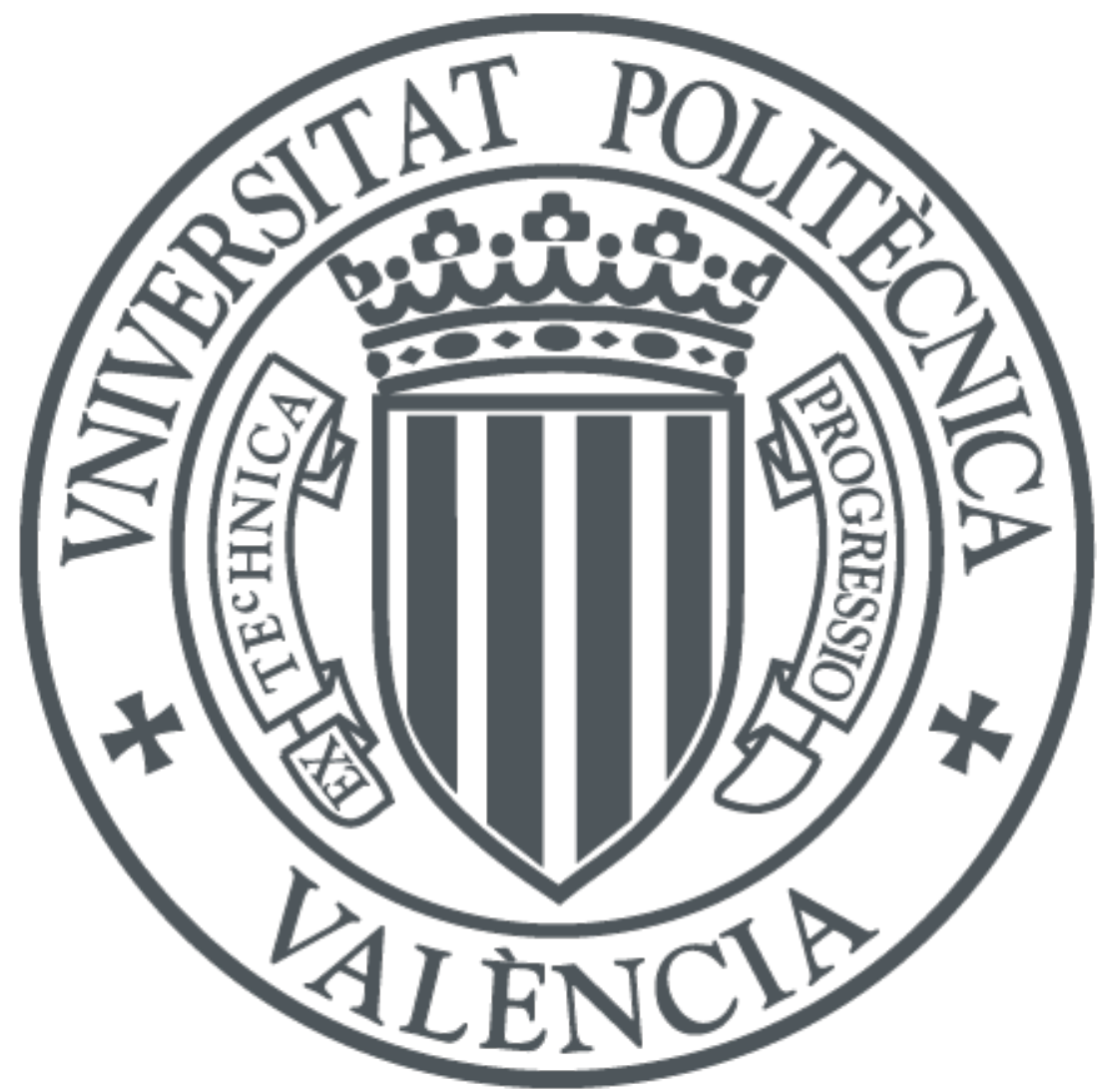

The final publication is available at

https://doi.org/10.1615/Int.J.UncertaintyQuantification.2020032659

Copyright Begell House

Additional Information 


\title{
VARIANCE REDUCTION METHODS AND
}

MULTILEVEL MONTE CARLO STRATEGY FOR

ESTIMATING DENSITIES OF SOLUTIONS TO

RANDOM SECOND ORDER LINEAR DIFFERENTIAL

\section{EQUATIONS}

\author{
Marc Jornet, ${ }^{1, *}{ }^{, *}$ Julia Calatayud, ${ }^{1}$ Olivier P. Le Maître, ${ }^{2} \mathcal{E}$ \\ Juan Carlos Cortés ${ }^{1}$ \\ ${ }^{1}$ Instituto de Matemática Multidisciplinar, Universitat Politècnica de València, Camí de Vera \\ s/n, 46022, Valencia, Spain \\ ${ }^{2}$ CMAP, CNRS, INRIA, École Polytechnique, Institut Polytechnique de Paris, 91128 Palaiseau, \\ France.
}

*Address all correspondence to: Marc Jornet, Instituto de Matemática Multidisciplinar, Universitat Politècnica de València, Camí de Vera s/n, 46022, Valencia, Spain, E-mail: marjorsa@doctor.upv.es

Original Manuscript Submitted: mm/dd/yyyy; Final Draft Received: mm/dd/yyyy

This paper concerns the estimation of the density function of the solution to a random non-autonomous second-order linear differential equation with analytic data processes. In a recent contribution, we proposed to express the density function as an expectation, and we used a standard Monte Carlo algorithm to approximate the expectation. Although the algorithms worked satisfactorily for most test-problems, some numerical challenges emerged for others, due to large statistical errors. In these situations, the convergence of the Monte Carlo simulation slows down severely, and noisy features plague the estimates. In this paper, we focus on computational aspects and propose several variance reduction methods to remedy these issues and speed up the convergence. First, we introduce a path-wise selection of the approximating processes which aims at controlling the variance of the estimator. Second, we propose a hybrid method, combining Monte Carlo and deterministic quadrature rules, to estimate the expectation. Third, we exploit the series expansions of the solutions to design a multilevel Monte Carlo estimator. The proposed methods are implemented and tested on several numerical examples to highlight the theoretical discussions and demonstrate the significant improvements achieved.

KEY WORDS: random linear differential equation; probability density function; standard and multilevel Monte Carlo simulation; analysis of algorithms 


\section{INTRODUCTION AND PRELIMINARIES}

This paper concerns the analysis of stochastic processes $X(t)$ solution to general random non-autonomous second order linear differential equations:

$$
\left\{\begin{array}{l}
\ddot{X}(t)+A(t) \dot{X}(t)+B(t) X(t)=0 \\
X\left(t_{0}\right)=Y_{0}, \dot{X}\left(t_{0}\right)=Y_{1}
\end{array}\right.
$$

The time-dependent coefficients $A(t)$ and $B(t)$ are stochastic processes and the initial conditions $Y_{0}$ and $Y_{1}$ are random variables. These random quantities are defined in an underlying complete probability space $(\Omega, \mathcal{F}, \mathbb{P})$, where $\Omega$ is the sample space formed by outcomes $\omega \in \Omega, \mathcal{F} \subseteq 2^{\Omega}$ is the $\sigma$-algebra of events, and $\mathbb{P}$ is the probability measure. Problem (1) belongs to a broader class of equations referred to as random differential equations [1-3]. Random differential equations are those in which random effects are manifested through input coefficients, initial condition and/or forcing term, in the form of random variables or regular stochastic processes with any type of probability distribution; the solution stochastic process is differentiable (not to be confused with stochastic differential equations of Itô type driven by irregular processes, whose solutions exhibit nondifferentiable sample paths; see [4, pp. 96-98] for the distinction between random and stochastic differential equations). Random differential equations are generally studied with two distinct approaches, based on random calculus and sample paths. For the necessary theory on random calculus, specifically mean square calculus with the norm $\|Z\|_{2}=\mathbb{E}\left[Z^{2}\right]^{1 / 2}$ for random variables $Z$ in the Lebesgue space $L^{2}(\Omega)$, we refer the reader to [1].

In this paper, we assume that $A(t)$ and $B(t)$ are analytic stochastic processes in the mean square sense [1, p. 99], so they can be expanded as

$$
A(t)=\sum_{n=0}^{\infty} A_{n}\left(t-t_{0}\right)^{n}, \quad B(t)=\sum_{n=0}^{\infty} B_{n}\left(t-t_{0}\right)^{n}, \quad t \in\left(t_{0}-r, t_{0}+r\right),
$$

where $A_{0}, A_{1}, \ldots, B_{0}, B_{1}, \ldots$ are second order random variables, which may or may not be statistically independent, given by $A_{i}=A^{(i)}\left(t_{0}\right) / i$ ! and $B_{i}=B^{(i)}\left(t_{0}\right) / i$ !, with the $i$-th derivative considered in the mean square sense. The Taylor series of $A(t)$ and $B(t)$ in (2) present mean square convergence. It is interesting to observe that the analytic property in the mean sense of a stochastic process is determined by the ordinary analytic property of its correlation function [1, Th. 4.4.3]. The regularity of $A(t)$ and $B(t)$ does not allow them to be Brownian motions or white noise processes, thus the distinct treatment of our problem compared to Itô calculus. 
The linear problem (1) with analytic data processes has raised interest in the literature on random differential equations. The study was initialized in particular equations from Mathematical Physics, such as Airy, Hermite and Legendre differential equations [5-7]. The main goal of these contributions was to construct the mean square analytic solution

$$
X(t)=\sum_{n=0}^{\infty} X_{n}\left(t-t_{0}\right)^{n}
$$

on $\left(t_{0}-r, t_{0}+r\right)$ via the Fröbenius method, thus obtaining approximations of the expectation and the variance of $X(t), \mathbb{E}[X(t)]$ and $\mathbb{V}[X(t)]$. Other techniques to study particular cases of (1) are PC expansions [8,9], homotopy method [10], variational iteration [11], Adomian decomposition [12], and differential transform method [13,14].

The general problem (1) was successfully solved in $[15,16]$ using the Fröbenius method. It was proved that, if the power series from (2) converge in $\mathrm{L}^{\infty}(\Omega)$ (with the essential supremum norm $\|\cdot\|_{\infty}$ ) and if $Y_{0}, Y_{1} \in \mathrm{L}^{2}(\Omega)$, then there is a unique mean square solution of the form (3). This result is extendable to a more general measure norm, in the Lebesgue space $\left(\mathrm{L}^{p}(\Omega),\|\cdot\|_{p}\right), 1 \leq p \leq \infty$ : if $Y_{0}, Y_{1} \in \mathrm{L}^{p}(\Omega)$, then the series (3) converges in $\mathrm{L}^{p}(\Omega)$ and solves (1) in the $\mathrm{L}^{p}(\Omega)$ sense. The convergence is exponentially fast, although not uniformly in $t$; it deteriorates when $t$ or the norms of the input coefficients grow.

Throughout the paper, we assume that the power series from (2) converge in $\mathrm{L}^{\infty}(\Omega)$ (with $A_{0}, A_{1}, \ldots, B_{0}, B_{1}, \ldots$ bounded) and that $Y_{0}, Y_{1} \in \mathrm{L}^{2}(\Omega)$, so that the conditions stated above for the existence of mean square stochastic solution are fulfilled.

The computation of the probability density function of $X(t)$ is a more ambitious goal for quantifying uncertainty. When $X(t)$ is absolutely continuous, meaning that its probability law is absolutely continuous with respect to the Lebesgue measure, then there exists a non-negative Borel measurable function $f_{X(t)}(x)=\frac{\mathrm{d}\left(\mathbb{P} \circ X(t)^{-1}\right)(x)}{\mathrm{d} x}$ characterized by $\mathbb{P}[X(t) \in \mathcal{C}]=\int_{\mathcal{C}} f_{X(t)}(x) \mathrm{d} x(\mathcal{C} \subseteq \mathbb{R}$ is any Borel set). Confidence intervals and statistical moments of the response $X(t)$ can be computed through the probability density function.

In random differential equation problems, the probability density function is usually computed through transformation of random variables [17-23]. This approach was used in [24] for the autonomous version of (1), when $A(t)=A$ and $B(t)=B$ are time-independent random variables. We extended the computation of the density function to the general problem (1) in [25].

The procedure presented in [25] to derive the probability density function $f_{X(t)}(x)$ is briefly summarized. Let $\left\{S_{0}(t), S_{1}(t)\right\}$ be the fundamental set of solutions to (1) satisfying the deterministic initial conditions $S_{0}\left(t_{0}\right)=1$, $\dot{S}_{0}\left(t_{0}\right)=0, S_{1}\left(t_{0}\right)=0$ and $\dot{S}_{1}\left(t_{0}\right)=1$. Both $S_{0}(t)$ and $S_{1}(t)$ are given by infinite random power series, converging 
1 in $\mathrm{L}^{\infty}(\Omega)$. The solution $X(t)$ is expressed as $X(t)=Y_{0} S_{0}(t)+Y_{1} S_{1}(t)$. If $S_{0}(t) \neq 0$ almost surely and $Y_{0}$ has a 2 density $f_{Y_{0}}$, we obtain

$$
f_{X(t)}(x)=\mathbb{E}\left[Z_{0}(x, t)\right]
$$

3 where

$$
Z_{0}(x, t)=f_{Y_{0}}\left(\frac{x-Y_{1} S_{1}(t)}{S_{0}(t)}\right) \frac{1}{\left|S_{0}(t)\right|}
$$

4 Similarly, if $S_{1}(t) \neq 0$ almost surely and $Y_{1}$ possesses a density $f_{Y_{1}}$, then

$$
f_{X(t)}(x)=\mathbb{E}\left[Z_{1}(x, t)\right]
$$

5 where

$$
Z_{1}(x, t)=f_{Y_{1}}\left(\frac{x-Y_{0} S_{0}(t)}{S_{1}(t)}\right) \frac{1}{\left|S_{1}(t)\right|}
$$

6 When a simple closed-form expression for $S_{0}(t)$ and $S_{1}(t)$ is not available, truncating the power series $S_{0}(t)$ and $S_{1}(t)$

7 to the $N \geq 0$ first terms is necessary. We denote $S_{0}^{N}(t)$ and $S_{1}^{N}(t)$, the partial sums which converge respectively to $8 S_{0}(t)$ and $S_{1}(t)$, in $\mathrm{L}^{\infty}(\Omega)$, as $N \rightarrow \infty$. The truncated series of $X(t)$ is given by $X^{N}(t)=Y_{0} S_{0}^{N}(t)+Y_{1} S_{1}^{N}(t)$, 9 which converges to $X(t)$ as $N \rightarrow \infty$ in the mean square sense. The probability density function of $X^{N}(t)$ is, whenever $S_{0}^{N}(t) \neq 0$ almost surely or $S_{1}^{N}(t) \neq 0$ almost surely,

$$
f_{X^{N}(t)}(x)=\mathbb{E}\left[Z_{0}^{N}(x, t)\right]
$$

11 or

$$
f_{X^{N}(t)}(x)=\mathbb{E}\left[Z_{1}^{N}(x, t)\right]
$$

12 where

$$
Z_{0}^{N}(x, t)=f_{Y_{0}}\left(\frac{x-Y_{1} S_{1}^{N}(t)}{S_{0}^{N}(t)}\right) \frac{1}{\left|S_{0}^{N}(t)\right|}, Z_{1}^{N}(x, t)=f_{Y_{1}}\left(\frac{x-Y_{0} S_{0}^{N}(t)}{S_{1}^{N}(t)}\right) \frac{1}{\left|S_{1}^{N}(t)\right|}
$$

Several theoretical results from [25] justify that $f_{X^{N}(t)}(x)$ tends to the target density function $f_{X(t)}(x)$ as $N \rightarrow \infty$ in a neighborhood of $t_{0}$. The convergence is pointwise and in $\mathrm{L}^{p}(\mathbb{R})$, for $1 \leq p<\infty$. Convergence in terms of the total variation and the Hellinger distances also holds. The hypotheses set to establish the convergence are mainly related to the continuity of $f_{Y_{0}}$ or $f_{Y_{1}}$. If $f_{Y_{0}}$ or $f_{Y_{1}}$ are Hölder continuous, then exponential convergence holds, albeit not uniform in $t$ and $x$. 
In [25], the expectation from (8) and (9) is approximated using Monte Carlo (MC) simulation, via an explicit algorithm. Either (8) or (9) are selected, and realizations of the involved random variables are generated to compute the sample average. This introduces an error due to sampling, apart from the bias error $\theta_{N}(x, t)=f_{X(t)}(x)-f_{X^{N}(t)}(x)$. The convergence rate of the MC procedure towards $f_{X^{N}(t)}(x)$ depends on the finite number of realizations and on the variances $\mathbb{V}\left[Z_{0}^{N}(x, t)\right], \mathbb{V}\left[Z_{1}^{N}(x, t)\right]$. If these variances are large or infinite, the convergence rate of the MC simulation deteriorates severely and noisy estimates of $f_{X^{N}(t)}(x)$ are produced, thus invalidating the results. This phenomenon was observed in the numerical experiments from [25, Example 5.3]. It is highly related to having small denominators $\left|S_{0}^{N}(t)\right|$ and $\left|S_{1}^{N}(t)\right|$ at certain realizable paths, as this may produce higher dispersion of $1 /\left|S_{0}^{N}(t)\right|$ and $1 /\left|S_{1}^{N}(t)\right|$

The main goal of this paper is to improve the algorithm from [25] via several variance reduction methods to achieve good convergence of the MC simulation and prevent noisy features. All the proposed methods are implemented and tested using the software Mathematica ${ }^{\circledR}$, version 11.2 [26].

In Section 2, we propose a path-wise selection of the initial condition to avoid the smallest denominator, $\left|S_{0}^{N}(t)\right|$ or $\left|S_{1}^{N}(t)\right|$, used in (4)-(6) . In this manner, we control the variance of the sampling error, and we improve the quality of the numerical results significantly. An important assumption to apply this selection is that the two initial conditions have probability density functions.

In Section 3, we improve further the algorithm of Section 2 with a deterministic quadrature to approximate the expectation with respect to $Y_{0}-Y_{1}$. It allows for a reduction of variance when $Y_{0}$ and $Y_{1}$ are responsible for most of the estimator variability (this can be checked using Sobol indices). A detailed comparison with the methodology of Section 2, in terms of variance reduction, density approximation, and error versus complexity, is carried out.

In Section 4, the MC approach from Section 2 is extended considering a multilevel strategy. Originally introduced in $[27,28]$, the idea of multilevel $\mathrm{MC}$ is to accelerate the convergence of the MC simulation by decomposing the target expectation into the sum of expectations of increments (through a telescopic sum identity) whose variances decay rapidly. Multilevel MC simulation is an improved sampling strategy that balances the bias and sampling errors, to obtain an overall reduction of the computational complexity compared to the standard MC algorithm. Multilevel sampling strategies have been employed for the approximation of statistics in stochastic differential equations and random partial differential equations, where the bias classically arises from a mesh discretization [29-31]. However, to our knowledge, the application to the density estimation of random differential equations solution is completely original.

Finally, Section 5 draws the main conclusions of the paper and discusses potential avenues for future research on 
18

algorithmic improvements, linear random differential equations, and density approximations.

$$
\text { The proofs of the mathematical results (propositions and lemmas) are postponed to the Appendix. }
$$

\section{VARIANCE REDUCTION BY PATH-WISE SELECTION OF THE INITIAL CONDITION USED IN THE DENSITY EXPRESSION}

In [25], the density function $f_{X^{N}(t)}(x)$ is approximated using a standard MC sampling strategy, by initially selecting either $Z_{0}^{N}(x, t)$ or $Z_{1}^{N}(x, t)$ (see (10)), and only one of these processes. The convergence rate of the sample average towards $\mathbb{E}\left[Z_{0}^{N}(x, t)\right]$ or $\mathbb{E}\left[Z_{1}^{N}(x, t)\right]$ depends on $\mathbb{V}\left[Z_{0}^{N}(x, t)\right]$ or $\mathbb{V}\left[Z_{1}^{N}(x, t)\right]$, respectively. If $\mathbb{V}\left[Z_{0}^{N}(x, t)\right]$ and $\mathbb{V}\left[Z_{1}^{N}(x, t)\right]$ are large or infinity, the convergence rate deteriorates severely and noisy features may plague the statistical approximation to $f_{X^{N}(t)}(x)$. This issue was observed in [25, Example 5.3].

In this section we propose a variance reduction method by combining the choices of $Z_{0}^{N}(x, t)$ and $Z_{1}^{N}(x, t)$. Consider one realization of $S_{0}^{N}(t)$ and $S_{1}^{N}(t)$. These realizations are computed recursively in the computer from realizations of $A_{0}, \ldots, A_{N-2}, B_{0}, \ldots, B_{N-2}$, see [25]. If $\left|S_{0}^{N}(t)\right| \geq\left|S_{1}^{N}(t)\right|$ then we pick $Z_{0}^{N}(x, t)$, and vice versa otherwise. In such a way, we avoid the smallest denominator in the expressions of $Z_{0}^{N}(x, t)$ and $Z_{1}^{N}(x, t)$.

We assume that $Y_{0}, Y_{1}$ and $\left(A_{0}, A_{1}, \ldots, B_{0}, B_{1}, \ldots\right)$ are independent. We also suppose that $Y_{0}$ and $Y_{1}$ are absolutely continuous random variables (otherwise this method is no longer applicable). Given the events $G_{0}(t)=$ $\left\{\left|S_{0}(t)\right| \geq\left|S_{1}(t)\right|\right\}, G_{1}(t)=\left\{\left|S_{1}(t)\right|>\left|S_{0}(t)\right|\right\}, G_{0}^{N}(t)=\left\{\left|S_{0}^{N}(t)\right| \geq\left|S_{1}^{N}(t)\right|\right\}$ and $G_{1}^{N}(t)=\left\{\left|S_{1}^{N}(t)\right|>\right.$ $\left.\left|S_{0}^{N}(t)\right|\right\}$, we define the random processes

$$
Z(x, t)=Z_{0}(x, t) 1_{G_{0}(t)}+Z_{1}(x, t) 1_{G_{1}(t)}
$$

$$
Z^{N}(x, t)=Z_{0}^{N}(x, t) 1_{G_{0}^{N}(t)}+Z_{1}^{N}(x, t) 1_{G_{1}^{N}(t)} .
$$

These processes correspond to path-wise selecting $f_{Y_{0}}$ or $f_{Y_{1}}$ according to $\left|S_{0}(t)\right| \geq\left|S_{1}(t)\right|$ or $\left|S_{1}(t)\right|>\left|S_{0}(t)\right|$ (in (11)), and $\left|S_{0}^{N}(t)\right| \geq\left|S_{1}^{N}(t)\right|$ or $\left|S_{1}^{N}(t)\right|>\left|S_{0}^{N}(t)\right|$ (in (12)). By path-wise selection, we refer to generate realizations of $S_{0}^{N}(t)$ and $S_{1}^{N}(t)$ (the paths/trajectories of the stochastic processes) and then select $Z_{0}^{N}(x, t)$ or $Z_{1}^{N}(x, t)$ accordingly. The density functions of $X^{N}(t)$ and $X(t)$ are then expressed as follows.

Proposition 1. The following relationships hold:

$$
f_{X(t)}(x)=\mathbb{E}[Z(x, t)], \quad f_{X^{N}(t)}(x)=\mathbb{E}\left[Z^{N}(x, t)\right]
$$

Volume x, Issue x, 2017 
1 where $Z(x, t)$ and $Z^{N}(x, t)$ are defined in (11) and (12).

2 The expectation $f_{X^{N}(t)}(x)=\mathbb{E}\left[Z^{N}(x, t)\right]$ may be approximated via a standard MC sampling strategy. A key 3 feature here is the uniform boundedness of $\mathbb{V}\left[Z^{N}(x, t)\right]$ with $N$, as shown in Proposition 3.

4 Lemma 2. Given any $T>t_{0}$, there exists a constant $\alpha>0$ such that

$$
\max \left\{\left|S_{0}(t)\right|,\left|S_{1}(t)\right|\right\} \geq 2 \alpha
$$

5 holds almost surely, for all $t \in\left[t_{0}, T\right]$. In particular, there exists an integer $N_{T, \alpha} \geq 0$ such that

$$
\max \left\{\left|S_{0}^{N}(t)\right|,\left|S_{1}^{N}(t)\right|\right\} \geq \alpha
$$

holds almost surely, for all $t \in\left[t_{0}, T\right]$ and $N \geq N_{T, \alpha}$.

Proposition 3. Suppose that $f_{Y_{0}}$ and $f_{Y_{1}}$ are bounded density functions. Given any $T>t_{0}$, the $\mathrm{L}^{2}(\Omega)$ norms of $Z(x, t)$ and $Z^{N}(x, t)$ are controlled as follows:

$$
\|Z(x, t)\|_{2} \leq \frac{\max \left\{\left\|f_{Y_{0}}\right\|_{\infty},\left\|f_{Y_{1}}\right\|_{\infty}\right\}}{2 \alpha}, \quad\left\|Z^{N}(x, t)\right\|_{2} \leq \frac{\max \left\{\left\|f_{Y_{0}}\right\|_{\infty},\left\|f_{Y_{1}}\right\|_{\infty}\right\}}{\alpha}
$$

for $x \in \mathbb{R}, t \in\left[t_{0}, T\right]$ and $N \geq N_{T, \alpha}$, where $\alpha$ is the constant from Lemma 2 .

As a consequence, the variance here is well controlled, in contrast with [25]. The estimate of $f_{X^{N}(t)}(x)=$ $\mathbb{E}\left[Z^{N}(x, t)\right]$ using MC simulation does not present convergence problems.

Remark 1. Lemma 2 is not only important from a numerical point of view, but also for the following theoretical fact. Our paper [25] justified the pointwise convergence of $\left\{f_{X^{N}(t)}(x)\right\}_{N=0}^{\infty}$ towards $f_{X(t)}(x)$ in a neighborhood of $t_{0}$. The neighborhood was constructed in such a way that $S_{0}(t)$ and/or $S_{1}(t)$ are greater than a positive constant almost surely (see [25, Remarks 3.5, 3.10]), so that the denominators from (10) are controlled. Lemma 2 shows that

$$
\frac{1}{\left|S_{0}(t)\right|} 1_{G_{0}(t)}+\frac{1}{\left|S_{1}(t)\right|} 1_{G_{1}(t)}=\frac{1}{\max \left\{\left|S_{0}(t)\right|,\left|S_{1}(t)\right|\right\}}
$$

is upper bounded by a finite constant almost surely, for every tin the domain of definition of (1). Hence, the theoretical results from [25] justifying the convergence of $f_{X^{N}(t)}(x)=\mathbb{E}\left[Z^{N}(x, t)\right]$ to $f_{X(t)}(x)=\mathbb{E}[Z(x, t)]$ as $N \rightarrow \infty$ do hold for every $t$ where $X(t)$ is well-defined, not just on a neighborhood of $t_{0}$. Notice that this statement requires both 
$Y_{0}$ and $Y_{1}$ to be absolutely continuous, and $Y_{0}, Y_{1}$ and $\left(A_{0}, A_{1}, \ldots, B_{0}, B_{1}, \ldots\right)$ to be independent. These are the hypotheses under which the methodology from this section is supported.

Given a time variable $t$, a point $x$ in the density domain of $X^{N}(t)$, a truncation order $N$ and a number $M$, the whole procedure described in this section is structured as follows:

\section{Procedure 1.}

Part 1 Generate $M$ realizations of $S_{0}^{N}(t)$ and $S_{1}^{N}(t)$

Part 2 For each realization, if $\left|S_{0}^{N}(t)\right| \geq\left|S_{1}^{N}(t)\right|$, set a realization of $Y_{1} \sim f_{Y_{1}}$ to obtain the realization of $Z^{N}(x, t)$ given by $Z_{0}^{N}(x, t)$ (see (10), (12)). Otherwise set a realization of $Y_{0} \sim f_{Y_{0}}$ to obtain the realization of $Z^{N}(x, t)$ given by $Z_{1}^{N}(x, t)$.

Part 3 Compute the sample mean of the $M$ realizations of $Z^{N}(x, t)$. This value estimates the expectation $f_{X^{N}(t)}(x)=$ $\mathbb{E}\left[Z^{N}(x, t)\right]$ with MC simulation. We denote the estimate (output function) by $f_{X}^{N, M}(x, t)$, which tends to $f_{X^{N}(t)}(x)$ as $M \rightarrow \infty$.

The complexity (or cost, or work, which is defined in this paper as the total number of arithmetic operations) of Procedure 1 is $\mathcal{O}\left(M N^{2}\right)$, in general. Recall that each realization of $S_{0}^{N}(t)$ and $S_{1}^{N}(t)$ is computed recursively with $N$ and requires $\mathcal{O}\left(N^{2}\right)$ operations [25].

As discussed in [25], when $A(t)$ and $B(t)$ are random polynomials instead of infinite random series, the cost per realization of $S_{0}^{N}(t)$ and $S_{1}^{N}(t)$ is reduced to $\mathcal{O}(N)$ operations. Then the global complexity of Procedure 1 is $\mathcal{O}(M N)$ only.

Obviously, in the case where $S_{0}(t)$ and $S_{1}(t)$ are known in closed-form expression (this occurs for simple problems (1), such as the autonomous case), Procedure 1 can be run with $N=\infty$, that is, by computing realizations of $S_{0}(t)$ and $S_{1}(t)$ directly. The complexity of the algorithm is $\mathcal{O}(M)$ in such a case. The error is statistical and only due to the finite sampling.

Procedure 1 may be run symbolically on the variable $x$, thus having a symbolic expression for the output $f_{X}^{N, M}(x, t)$ in the end. The algorithm from [25] was run symbolically. However, symbolic computations do not allow a large number of simulations $M$. Thus, in cases where $M$ needs to be large enough to achieve convergence, we recommend to discretize $x$ in a domain of interest for the target density.

In the following two examples, we illustrate and assess the theoretical discussion from this section. The main goal is to highlight the improvement of Procedure 1 compared with [25], in terms of variance reduction of the MC 
estimator and accuracy of the density estimation. The first example, Example 1, focuses on a simple autonomous problem with exact solution, so that $N=\infty$. The second example, Example 2, deals with a more complex problem, where $A(t)$ and $B(t)$ are one-degree random polynomials and the series solution is truncated to a finite number $N$ of terms.

Example 1. We start by considering a very simple problem (1):

$$
\left\{\begin{array}{l}
\ddot{X}(t)+B^{2} X(t)=0, t \in \mathbb{R}, \\
X\left(t_{0}=0\right)=Y_{0}, \dot{X}\left(t_{0}=0\right)=Y_{1} .
\end{array}\right.
$$

Despite its simplicity, (15) is a useful example to illustrate the theoretical discussion from this section.

The following probability distributions are considered: $B \sim \operatorname{Uniform}(1,2.5), Y_{0}, Y_{1} \sim \operatorname{Normal}(1, \sigma=0.1)$, all of them independent. As $B$ is bounded and the initial conditions $Y_{0}$ and $Y_{1}$ have absolute moments of any order, there exists an analytic solution $X(t)$ in the $\mathrm{L}^{p}(\Omega)$ sense, for $1 \leq p<\infty$. The fundamental system is explicitly known: $S_{0}(t)=\cos (B t), S_{1}(t)=\sin (B t) / B, t \in \mathbb{R}$. Thus, Procedure 1 can be applied with $N=\infty$, by taking realizations from $S_{0}(t)$ and $S_{1}(t)$ directly. We consider time $t=10$, and the goal is to approximate the density function $f_{X(t=10)}(x)$ using MC methods.

In Figure 1, left panel, we estimate $\mathbb{E}\left[Z_{0}(x, t=10)\right]$ and $\mathbb{E}\left[Z_{1}(x, t=10)\right]$ with the MC procedure from [25], by generating $M=100,000$ realizations of the random inputs. These expectations correspond to $f_{X(t=10)}(x)$. Observe that noisy features plague the estimates of the density function. In Figure 1, right panel, we plot estimates of the variances $\mathbb{V}\left[Z_{0}(x, t=10)\right]$ and $\mathbb{V}\left[Z_{1}(x, t=10)\right]$. Their large values slow down the convergence of the MC simulation, thus explaining the noisy behavior of the estimates in the left plot. In fact, at the points $x$ where the variance is larger, more noise is perceived in the estimate of the expectation.

In Figure 2, left panel, we approximate $f_{X(t=10)}(x)$ via $\mathbb{E}[Z(x, t=10)]$, by using the proposed Procedure 1 with $M=100,000$ realizations. Observe that now the estimate is smooth, thus resolving the convergence challenge. In Figure 2, right panel, we depict an estimate for $\mathbb{V}[Z(x, t=10)]$. This variance is shown to be small compared to $\mathbb{V}\left[Z_{0}(x, t=10)\right]$ and $\mathbb{V}\left[Z_{1}(x, t=10)\right]$ in Figure 1, thus allowing for faster convergence of the MC simulation. Hence, Procedure 1 improves [25] significantly.

It is interesting to observe the different behaviors of $Z_{0}(x, 10), Z_{1}(x, 10)$ and $Z(x, 10)=Z_{0}(x, 10) 1_{G_{0}(10)}+$ $Z_{1}(x, 10) 1_{G_{1}(10)}$ as real functions of $B, Y_{0}$ and $Y_{1}$. Figure 3 depicts the 3D graphs of the real functions $Z_{0}(0,10)$, $Z_{1}(0,10), Z_{0}(0,10) 1_{G_{0}(10)}$ and $Z_{1}(0,10) 1_{G_{1}(10)}$ (obviously, any other point $x$ different from 0 can also be taken). The 


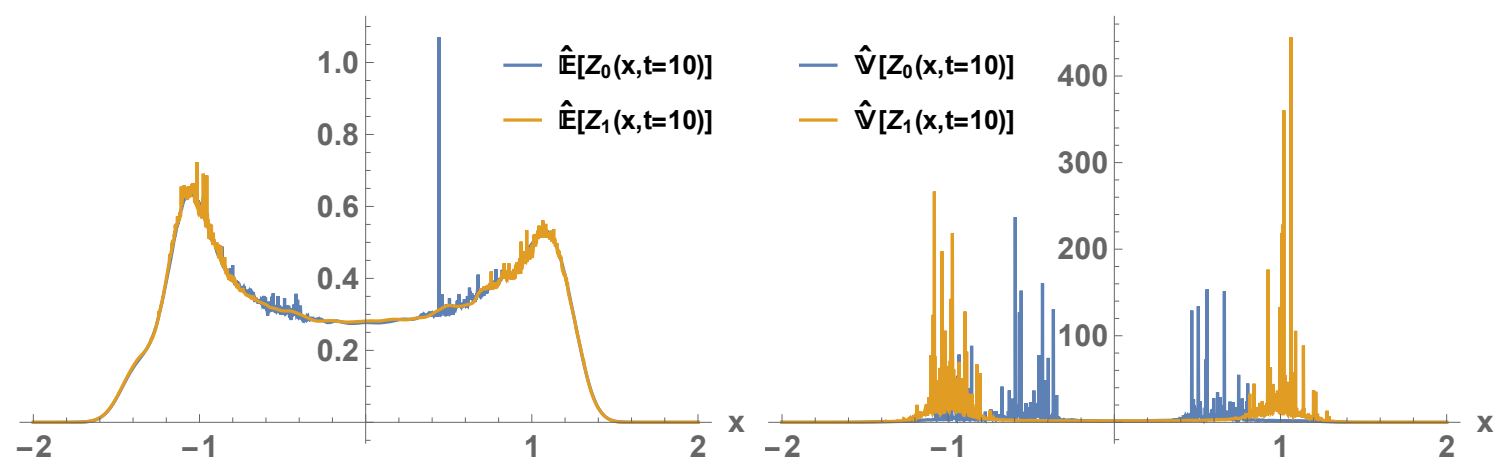

FIG. 1: Left: approximations of $f_{X(t=10)}(x)$ using estimates $\hat{\mathbb{E}}\left[Z_{0}(x, 10)\right]$ and $\hat{\mathbb{E}}\left[Z_{1}(x, 10)\right]$. Right: estimates $\hat{\mathbb{V}}\left[Z_{0}(x, 10)\right]$ and $\hat{\mathbb{V}}\left[Z_{1}(x, 10)\right]$. This figure corresponds to Example 1 .

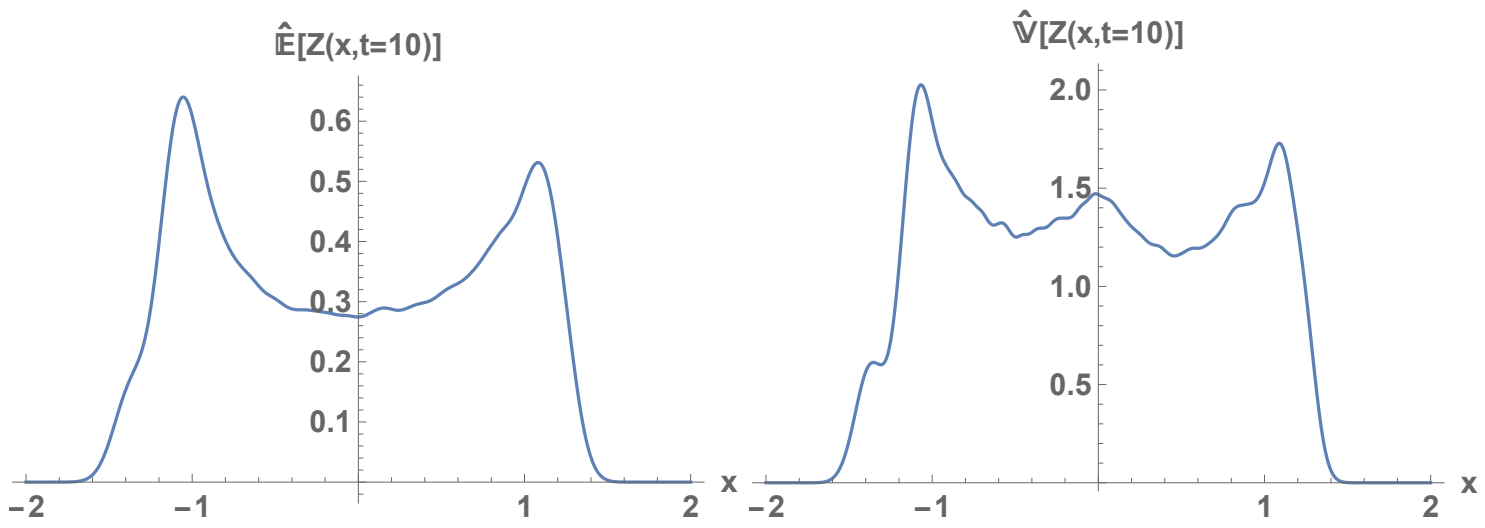

FIG. 2: Left: approximation of $f_{X(t=10)}(x)$ using estimate $\hat{\mathbb{E}}[Z(x, 10)]$. Right: estimate $\hat{\mathbb{V}}[Z(x, 10)]$. This figure corresponds to Example 1. 
functions $Z_{0}(0,10)$ and $Z_{1}(0,10)$ have singularities (because of vanishing denominators $|\cos (10 B)|$ and $\left.|\sin (10 B) / B|\right)$ and are strongly peaked. On the contrary, although $Z_{0}(0,10) 1_{G_{0}(10)}$ and $Z_{1}(0,10) 1_{G_{1}(10)}$ possess discontinuity points, their peaks are not that sharp. As we are getting rid of small denominators, the sharp peaks are avoided.
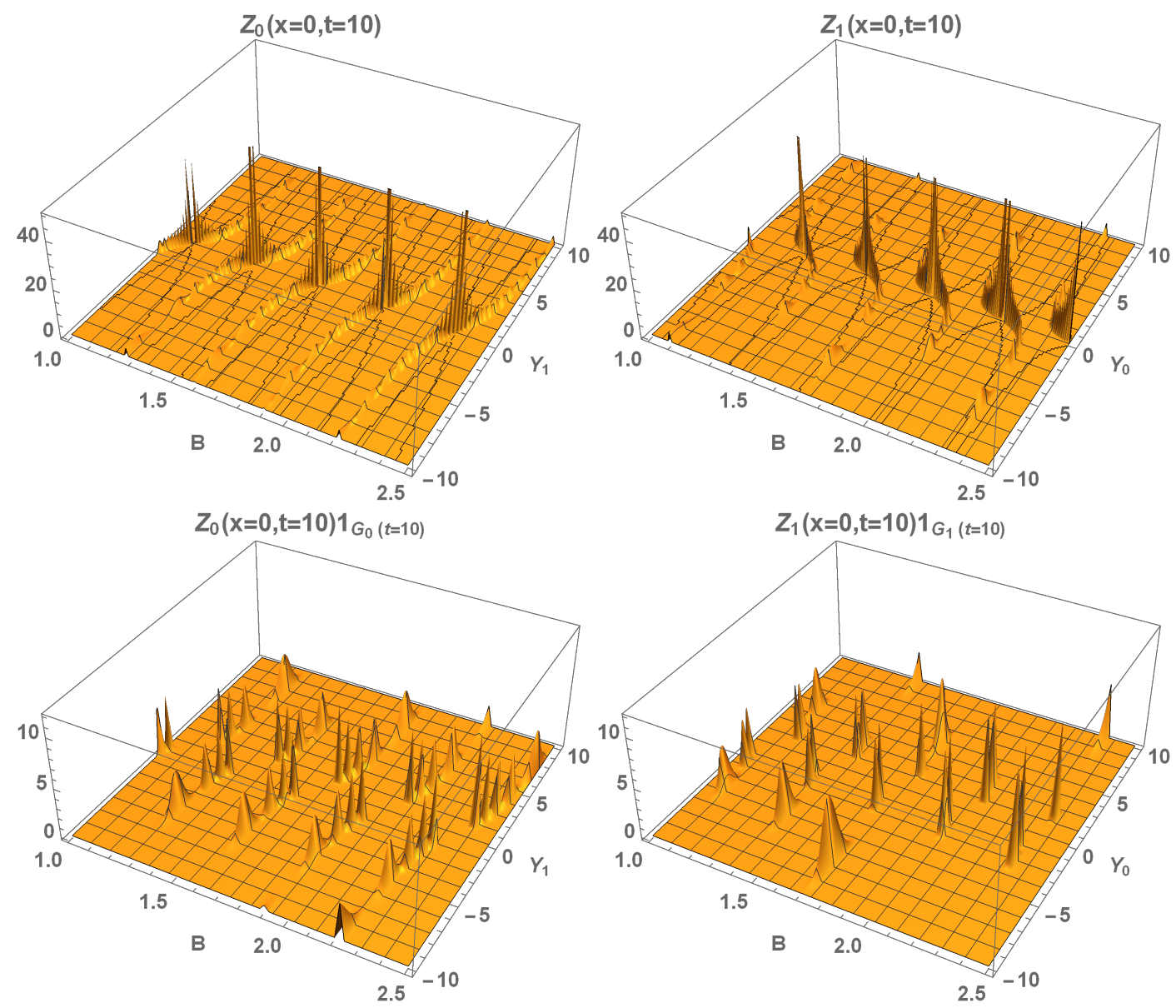

FIG. 3: 3D plots of the integrands $Z_{0}(0,10), Z_{1}(0,10)$ (their vertical axes have been restricted to $\left.[0,50]\right), Z_{0}(0,10) 1_{G_{0}(10)}$ and $Z_{1}(0,10) 1_{G_{1}(10)}$. This figure corresponds to Example 1 .

4 Example 2. We consider a more complex example now, based on degree one polynomial coefficients. Let

$$
\left\{\begin{array}{l}
\ddot{X}(t)+\left(A_{0}+A_{1} t\right) \dot{X}(t)+\left(B_{0}+B_{1} t\right) X(t)=0, t \in \mathbb{R} \\
X\left(t_{0}=0\right)=Y_{0}, \dot{X}\left(t_{0}=0\right)=Y_{1}
\end{array}\right.
$$

5 where $A_{0}=4, A_{1} \sim \operatorname{Uniform}(0,1),\left.B_{0} \sim \operatorname{Gamma}(2,2)\right|_{[0,4]}, B_{1} \sim \operatorname{Bernoulli}(0.35)$ and $Y_{0}, Y_{1} \sim \operatorname{Normal}(2,1)$, all 6 of them independent. Since the random coefficients $A_{0}, A_{1}, B_{0}$ and $B_{1}$ are bounded and the initial conditions $Y_{0}$ and 
$Y_{1}$ have finite absolute moments, there is an analytic solution $X(t)$ in the $\mathrm{L}^{p}(\Omega)$ sense, $1 \leq p<\infty$.

According to [25], the probability density function $f_{X(t)}(x)$ can be approximated pointwise using $f_{X^{N}(t)}(x)$ (with exponential convergence with $N$ because $f_{Y_{0}}$ and $f_{Y_{1}}$ are Lipschitz continuous on $\mathbb{R}$ ). We focus on the order of truncation $N=10$ to highlight the improvement of Procedure 1 compared with [25]. We estimate $f_{X^{N=10}(t=1.5)}(x)$ with both approaches.

In Figure 4, left panel, we estimate $\mathbb{E}\left[Z_{0}^{N=10}(x, t=1.5)\right]$ and $\mathbb{E}\left[Z_{1}^{N=10}(x, t=1.5)\right]$ with the algorithm described in [25]. With $M=20,000$ realizations, we observe noise in both estimates. This phenomenon is accurately explained by the plots of $\mathbb{V}\left[Z_{0}^{N=10}(x, t=1.5)\right]$ and $\mathbb{V}\left[Z_{1}^{N=10}(x, t=1.5)\right]$ in the right panel. It is very interesting to observe that the points $x$ where the variances are larger correspond to higher noise in the left plot. Indeed, large variance affects the rate of convergence of the MC simulation severely.
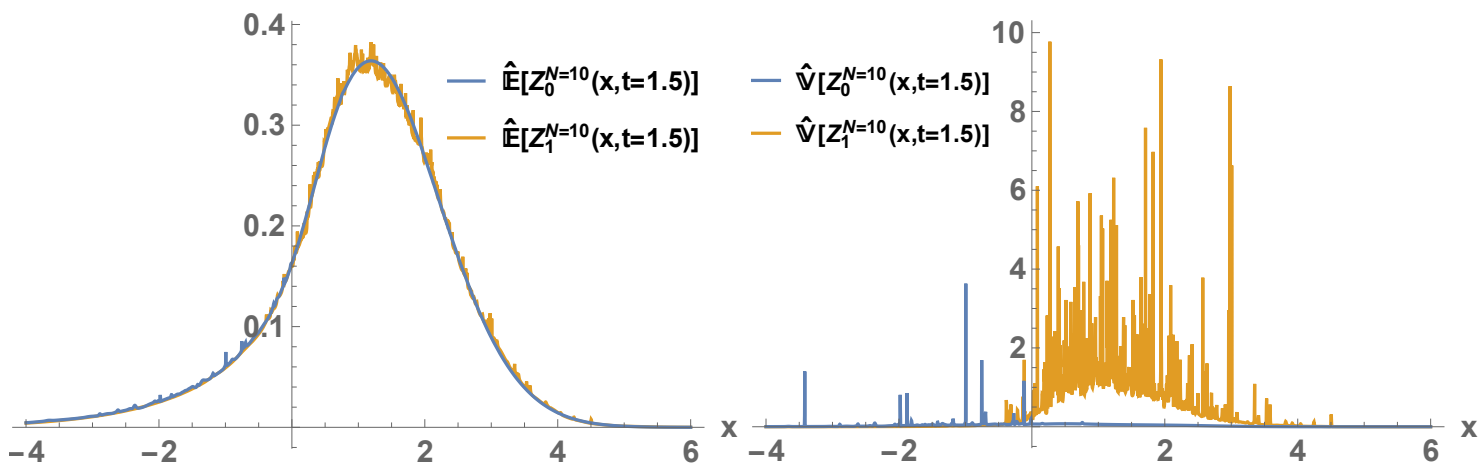

FIG. 4: Left: approximations of $f_{X^{N=10}(t=1.5)}(x)$ using estimates $\hat{\mathbb{E}}\left[Z_{0}^{N=10}(x, 1.5)\right]$ and $\hat{\mathbb{E}}\left[Z_{1}^{N=10}(x, 1.5)\right]$. Right: estimates $\hat{\mathbb{V}}\left[Z_{0}^{N=10}(x, 1.5)\right]$ and $\hat{\mathbb{V}}\left[Z_{1}^{N=10}(x, 1.5)\right]$. This figure corresponds to Example 2.

Procedure 1 has been designed to fix this issue. Figure 5, left panel, shows the smooth estimate of $f_{X^{N=10}(t=1.5)}(x)$ with $\mathbb{E}\left[Z^{N=10}(x, t=1.5)\right]$ and $M=20,000$ realizations. This good convergence is due to the small variance $\mathbb{V}\left[Z^{N=10}(x, t=1.5)\right]$ compared to $\mathbb{V}\left[Z_{0}^{N=10}(x, t=1.5)\right]$ and $\mathbb{V}\left[Z_{1}^{N=10}(x, t=1.5)\right]$, see the right panel of Figure 5. Hence, Procedure 1 shows significantly superior to a mere MC approach as in [25].

\section{VARIANCE REDUCTION BY USING QUADRATURE RULE FOR THE EXPECTATION WITH RESPECT TO THE INITIAL CONDITIONS}

By Proposition 1, the probability density function of the truncated series $X^{N}(t)$ is expressed as $f_{X^{N}(t)}(x)=$ $\mathbb{E}\left[Z^{N}(x, t)\right]$, where $Z^{N}(x, t)$ was defined in (12). The randomness in $Z^{N}(x, t)$ comes from the initial conditions, $Y_{0}$ and $Y_{1}$, and from the truncated series from the fundamental set, $S_{0}^{N}(t)$ and $S_{1}^{N}(t)$. An MC sampling procedure 


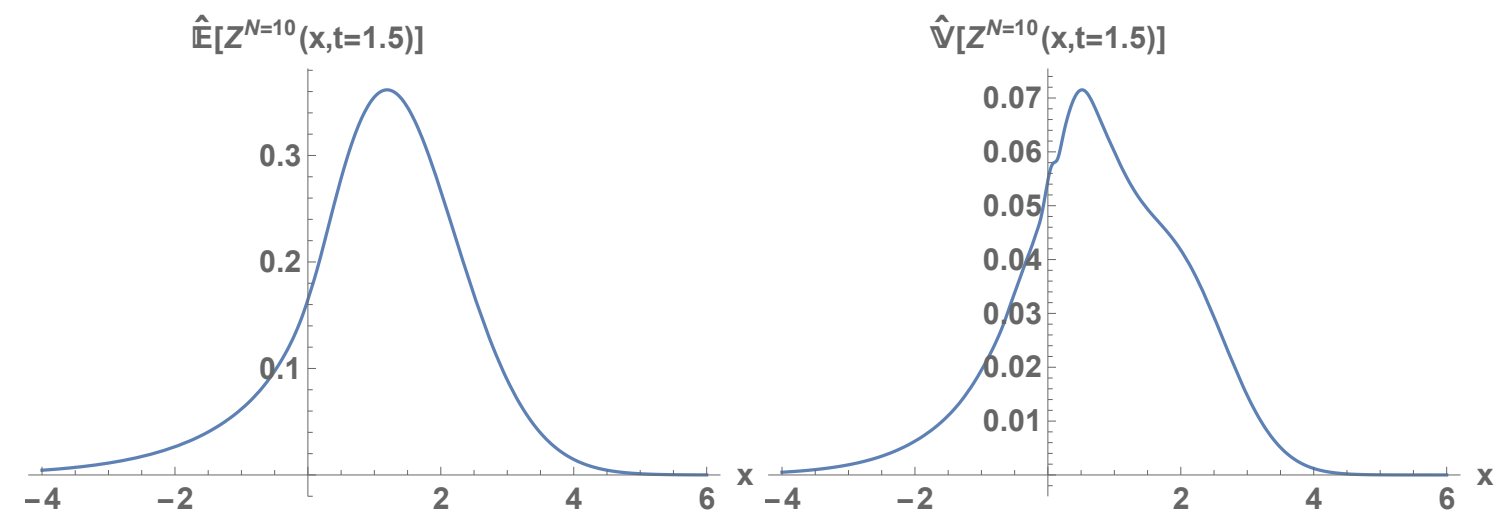

FIG. 5: Left: approximation of $f_{X^{N=10}(1.5)}(x)$ using estimate $\hat{\mathbb{E}}\left[Z^{N=10}(x, 1.5)\right]$. Right: estimate $\hat{\mathbb{V}}\left[Z^{N=10}(x, 1.5)\right]$. This figure corresponds to Example 2.

1

where $\left\{\left(s_{0, i}^{N}, s_{1, i}^{N}\right)\right\}_{i=1}^{M}$ are $M$ independent realizations of $\left(S_{0}^{N}(t), S_{1}^{N}(t)\right)$.

The variance of the sampling error from the approximation (17) is always smaller than in the standard MC approach from Procedure 1. Indeed, by the law of total variance,

$$
\begin{aligned}
\mathbb{V}\left[\mathbb{E}\left[Z^{N}(x, t) \mid S_{0}^{N}(t), S_{1}^{N}(t)\right]\right] & =\mathbb{V}\left[Z^{N}(x, t)\right]-\mathbb{E}\left[\mathbb{V}\left[Z^{N}(x, t) \mid S_{0}^{N}(t), S_{1}^{N}(t)\right]\right] \\
& \leq \mathbb{V}\left[Z^{N}(x, t)\right] .
\end{aligned}
$$

11 Equality holds only when $\mathbb{V}\left[Z^{N}(x, t) \mid S_{0}^{N}(t), S_{1}^{N}(t)\right]=0$. Essentially, using (17) we gain more reduction in the 
7 where

9

10

The influence of the variability of $Y_{0}, Y_{1}, S_{0}^{N}(t)$ and $S_{1}^{N}(t)$ on $Z^{N}(x, t)$ (variance-based sensitivity analysis) can be assessed using Sobol indices [32,33]. The random process $Z^{N}=Z^{N}(x, t)$ has the following orthogonal decomposition:

$$
Z^{N}=\mathbb{E}\left[Z^{N}\right]+\tilde{Z}_{S_{0}^{N}(t), S_{1}^{N}(t)}^{N}+\tilde{Z}_{Y_{0}, Y_{1}}^{N}+\tilde{Z}_{S_{0}^{N}(t), S_{1}^{N}(t), Y_{0}, Y_{1}}^{N}
$$

$$
\tilde{Z}_{S_{0}^{N}(t), S_{1}^{N}(t)}^{N}=Z_{S_{0}^{N}(t), S_{1}^{N}(t)}^{N}-\mathbb{E}\left[Z_{S_{0}^{N}(t), S_{1}^{N}(t)}^{N}\right], Z_{S_{0}^{N}(t), S_{1}^{N}(t)}^{N}=\mathbb{E}\left[Z^{N} \mid S_{0}^{N}(t), S_{1}^{N}(t)\right],
$$

$$
\tilde{Z}_{Y_{0}, Y_{1}}^{N}=Z_{Y_{0}, Y_{1}}^{N}-\mathbb{E}\left[Z_{Y_{0}, Y_{1}}^{N}\right], Z_{Y_{0}, Y_{1}}^{N}=\mathbb{E}\left[Z^{N} \mid Y_{0}, Y_{1}\right]
$$

$$
\tilde{Z}_{S_{0}^{N}(t), S_{1}^{N}(t), Y_{0}, Y_{1}}^{N}=Z_{S_{0}^{N}(t), S_{1}^{N}(t), Y_{0}, Y_{1}}^{N}-\mathbb{E}\left[Z_{S_{0}^{N}(t), S_{1}^{N}(t), Y_{0}, Y_{1}}^{N}\right]
$$

$$
Z_{S_{0}^{N}(t), S_{1}^{N}(t), Y_{0}, Y_{1}}^{N}=Z^{N}-\tilde{Z}_{S_{0}^{N}(t), S_{1}^{N}(t)}^{N}-\tilde{Z}_{Y_{0}, Y_{1}}^{N}
$$

Applying squared $\mathrm{L}^{2}(\Omega)$ norms, we derive the ANOVA-Hoeffding decomposition of the variance of $Z^{N}(x, t)$ :

$$
\mathbb{V}\left[Z^{N}\right]=\mathbb{V}\left[Z_{S_{0}^{N}(t), S_{1}^{N}(t)}^{N}\right]+\mathbb{V}\left[Z_{Y_{0}, Y_{1}}^{N}\right]+\mathbb{V}\left[Z_{S_{0}^{N}(t), S_{1}^{N}(t), Y_{0}, Y_{1}}^{N}\right]
$$

where $\mathbb{V}\left[Z_{S_{0}^{N}(t), S_{1}^{N}(t)}^{N}\right], \mathbb{V}\left[Z_{Y_{0}, Y_{1}}^{N}\right]$ and $\mathbb{V}\left[Z_{S_{0}^{N}(t), S_{1}^{N}(t), Y_{0}, Y_{1}}^{N}\right]$ measure the contribution to $Z^{N}(x, t)$ of $\left(S_{0}^{N}(t), S_{1}^{N}(t)\right)$ alone, $\left(Y_{0}, Y_{1}\right)$ alone, and the interactions between them, respectively. The standardization of the variance decomposition gives rise to the Sobol indices:

$$
\mathbb{S}_{S_{0}^{N}(t), S_{1}^{N}(t)}^{N}=\frac{\mathbb{V}\left[Z_{S_{0}^{N}(t), S_{1}^{N}(t)}^{N}\right]}{\mathbb{V}\left[Z^{N}\right]}, \quad \mathbb{S}_{Y_{0}, Y_{1}}^{N}=\frac{\mathbb{V}\left[Z_{Y_{0}, Y_{1}}^{N}\right]}{\mathbb{V}\left[Z^{N}\right]},
$$

$$
\mathbb{S}_{S_{0}^{N}(t), S_{1}^{N}(t), Y_{0}, Y_{1}}^{N}=\frac{\mathbb{V}\left[Z_{S_{0}^{N}(t), S_{1}^{N}(t), Y_{0}, Y_{1}}^{N}\right]}{\mathbb{V}\left[Z^{N}\right]},
$$

which are scaled in $[0,1]$. When $\mathbb{S}_{Y_{0}, Y_{1}}^{N}$ is near 1 , we expect more variance reduction in the MC estimate (17). 
Fix the index of the realizations $i=1, \ldots, M$. If $\left|s_{0, i}^{N}\right| \geq\left|s_{1, i}^{N}\right|$, then

$$
\begin{gathered}
\mathbb{E}\left[Z^{N}(x, t) \mid S_{0}^{N}(t)=s_{0, i}^{N}, S_{1}^{N}(t)=s_{1, i}^{N}\right]=\mathbb{E}\left[Z_{0}^{N}(x, t) \mid S_{0}^{N}(t)=s_{0, i}^{N}, S_{1}^{N}(t)=s_{1, i}^{N}\right] \\
=\frac{1}{\left|s_{0, i}^{N}\right|} \int_{\mathcal{S}_{Y_{1}}} f_{Y_{0}}\left(\frac{x-y_{1} s_{1, i}^{N}}{s_{0, i}^{N}}\right) f_{Y_{1}}\left(y_{1}\right) \mathrm{d} y_{1},
\end{gathered}
$$

where $\mathcal{S}_{Y_{1}}$ denotes the support of the random variable $Y_{1}$. If $\left|s_{1, i}^{N}\right|>\left|s_{0, i}^{N}\right|$, then

$$
\begin{gathered}
\mathbb{E}\left[Z^{N}(x, t) \mid S_{0}^{N}(t)=s_{0, i}^{N}, S_{1}^{N}(t)=s_{1, i}^{N}\right]=\mathbb{E}\left[Z_{1}^{N}(x, t) \mid S_{0}^{N}(t)=s_{0, i}^{N}, S_{1}^{N}(t)=s_{1, i}^{N}\right] \\
=\frac{1}{\left|s_{1, i}^{N}\right|} \int_{\mathcal{S}_{Y_{0}}} f_{Y_{1}}\left(\frac{x-y_{0} s_{0, i}^{N}}{s_{1, i}^{N}}\right) f_{Y_{0}}\left(y_{0}\right) \mathrm{d} y_{0},
\end{gathered}
$$

being $\mathcal{S}_{Y_{0}}$ the support of $Y_{0}$. These integrals (21), (22), can be approximated using numerical integration schemes. For instance, Gaussian quadrature rules [9, pp. 39-41] are appropriate since the density functions $f_{Y_{1}}\left(y_{1}\right)$ and $f_{Y_{0}}\left(y_{0}\right)$

act as integration weights. In the case of (21) (the case of (22) is analogous), one considers orthogonal polynomials $\left\{P_{j}\left(y_{1}\right)\right\}_{j=0}^{\infty}$ (the degree of $P_{j}$ is $j$ ) with respect to the inner product of $\mathrm{L}^{2}\left(\mathcal{S}_{Y_{1}}\right)$ with weight function $f_{Y_{1}}\left(y_{1}\right)$. For example, if $Y_{1}$ has a Normal or Uniform distribution, then $\left\{P_{j}\left(y_{1}\right)\right\}_{j=0}^{\infty}$ corresponds to the family of Hermite and Legendre polynomials, respectively. In other cases where $Y_{1}$ does not possess a standard probability distribution from the Askey scheme, $\left\{P_{j}\left(y_{1}\right)\right\}_{j=0}^{\infty}$ may be constructed through a Gram-Schmidt orthogonalization procedure from the canonical basis $\left\{1, y_{1}, y_{1}^{2}, y_{1}^{3}, \ldots\right\}$. The integral (21) is approximated as follows:

$$
\frac{1}{\left|s_{0, i}^{N}\right|} \int_{\mathcal{S}_{Y_{1}}} f_{Y_{0}}\left(\frac{x-y_{1} s_{1, i}^{N}}{s_{0, i}^{N}}\right) f_{Y_{1}}\left(y_{1}\right) \mathrm{d} y_{1} \approx \frac{1}{\left|s_{0, i}^{N}\right|} \sum_{j=1}^{Q} w_{1, j}^{(Q)} f_{Y_{0}}\left(\frac{x-y_{1, j}^{(Q)} s_{1, i}^{N}}{s_{0, i}^{N}}\right) .
$$

Here, $Q$ is the finite degree of the quadrature, the nodes $\left\{y_{1, j}\right\}_{j=1}^{Q}$ are the zeros of $P_{Q}\left(y_{1}\right)$, and $\left\{w_{1, j}^{(Q)}\right\}_{j=1}^{Q}$ are the weights so that the integration rule is exact for polynomials of degree less than or equal to $2 Q-1$

The nodes $\left\{y_{0, j}\right\}_{j=1}^{Q},\left\{y_{1, j}\right\}_{j=1}^{Q}$, and the weights $\left\{w_{0, j}^{(Q)}\right\}_{j=1}^{Q},\left\{w_{1, j}^{(Q)}\right\}_{j=1}^{Q}$, only depend on $f_{Y_{0}}$ and $f_{Y_{1}}$, therefore they are independent of the realization $\left(s_{0, i}^{N}, s_{1, i}^{N}\right)$, the number of simulations $M$ and the truncation order $N$. Hence, the nodes and the weights should always be computed once for all at the beginning.

As more regularity we demand on $f_{Y_{0}}$ and $f_{Y_{1}}$, faster convergence the quadrature rule will achieve with $Q$. The extreme case appears when $f_{Y_{0}}$ and $f_{Y_{1}}$ are analytic real functions: the quadrature rule converges exponentially with $Q$ and therefore the bias error $\theta_{N}(x, t)=f_{X(t)}(x)-f_{X^{N}(t)}(x)$ is not seriously affected by the deterministic error arisen from the finite degree $Q$. Nonetheless, numerical problems in the quadrature approximations may arise when 
the integrands are strongly peaked. In this case, the degree $Q$ must be taken larger, otherwise the bias error may increase dramatically.

The procedure is described in the following steps:

\section{Procedure 2.}

Part 1 Generate $M$ realizations of $S_{0}^{N}(t)$ and $S_{1}^{N}(t)$.

Part 2 For each realization, if $\left|S_{0}^{N}(t)\right| \geq\left|S_{1}^{N}(t)\right|$, consider the realization $\mathbb{E}\left[Z^{N}(x, t) \mid S_{0}^{N}(t), S_{1}^{N}(t)\right]$ approximated by the quadrature $\left.\sum_{j=1}^{Q} w_{1, j}^{(Q)} Z_{0}^{N}(x, t)\right|_{Y_{1}=y_{1, j}^{(Q)}}$ (see (10), (12), (21), (23)). Otherwise consider the realization $\mathbb{E}\left[Z^{N}(x, t) \mid S_{0}^{N}(t), S_{1}^{N}(t)\right]$ approximated by $\left.\sum_{j=1}^{Q} w_{0, j}^{(Q)} Z_{1}^{N}(x, t)\right|_{Y_{0}=y_{0, j}^{(Q)}}$.

Part 3 Compute the sample mean of the $M$ realizations of $\mathbb{E}\left[Z^{N}(x, t) \mid S_{0}^{N}(t), S_{1}^{N}(t)\right]$. This value estimates the expectation $f_{X^{N}(t)}(x)=\mathbb{E}\left[Z^{N}(x, t)\right]=\mathbb{E}\left[\mathbb{E}\left[Z^{N}(x, t) \mid S_{0}^{N}(t), S_{1}^{N}(t)\right]\right]$ with MC simulation. We denote the estimate (output function) by $f_{X}^{N, M, Q}(x, t)$.

The complexity of Procedure 2 is $\mathcal{O}\left(M\left(N^{2}+Q\right)\right)$, in general. Recall that one realization of $S_{0}^{N}(t)$ and $S_{1}^{N}(t)$ requires $\mathcal{O}\left(N^{2}\right)$ operations when $A(t)$ and $B(t)$ are given by infinite random expansions. The quadrature rule demands $\mathcal{O}(Q)$ operations.

When $A(t)$ and $B(t)$ are random polynomials, one realization of $S_{0}^{N}(t)$ and $S_{1}^{N}(t)$ requires $\mathcal{O}(N)$ operations only. The complexity of the whole algorithm thus becomes $\mathcal{O}(M(N+Q))$.

In the case of simple problems (1) in which $S_{0}(t)$ and $S_{1}(t)$ are exactly known, Procedure 2 is run with $N=\infty$, that is, by computing exact realizations of $S_{0}(t)$ and $S_{1}(t)$. The error in this case comes from the MC simulation and the finite degree of the quadrature rule. The total complexity is given by $\mathcal{O}(M Q)$.

We finish this section with numerical experiments concerning the methodology and Procedure 2. In the following three examples, we will compare Procedure 1 and Procedure 2 in terms of variance reduction, density approximation and error against complexity. The first two examples, Example 3 and Example 4, deal with problems having closedform expressions for the fundamental sets $(N=\infty)$. In Example 3, the initial conditions $Y_{0}$ and $Y_{1}$ have large dispersion compared to $S_{0}(t)$ and $S_{1}(t)$ (this is checked using Sobol indices), so a significant variance reduction of the MC estimator is achieved by employing quadrature rules with respect to $f_{Y_{0}}$ and $f_{Y_{1}}$. On the contrary, Example 4 presents two initial conditions with small dispersion compared to $S_{0}(t)$ and $S_{1}(t)$, hence the crude MC approach from Procedure 1 is more efficient. The third example, Example 5, deals with orders of truncation $N<\infty$, which implies that computing the fundamental set $\left\{S_{0}(t), S_{1}(t)\right\}$ of the problem takes certain computational burden. This entails 
that, albeit the initial conditions have small dispersion compared to $S_{0}(t)$ and $S_{1}(t)$, both Procedure 1 and Procedure 2 present similar efficiency. After the three examples, we will conclude the section with specific recommendations regarding the use of Procedure 1 or Procedure 2.

Example 3. Consider (15) with input data $B \sim \operatorname{Uniform}(1,1.01)$ and $Y_{0}, Y_{1} \sim \operatorname{Normal}(1, \sigma=2)$, all of them independent. As the fundamental set $\left\{S_{0}(t), S_{1}(t)\right\}$ is known with closed-form expressions, $S_{0}(t)=\cos (B t)$ and $S_{1}(t)=\sin (B t) / B$, we take $N=\infty$, by sampling from $S_{0}(t)$ and $S_{1}(t)$ directly.

Here the randomness mainly comes from the initial conditions. This is checked using the Sobol indices from (19) and (20). As proposed in [34,35], the Sobol indices are computable using MC simulation with the formulas

$$
\mathbb{V}\left[Z_{S_{0}(t), S_{1}(t)}\right] \approx \frac{1}{M} \sum_{i=1}^{M} Z\left(s_{i}^{I}, y_{i}^{I}\right) Z\left(s_{i}^{I}, y_{i}^{I I}\right)-\frac{1}{M} \sum_{i=1}^{M} Z\left(s_{i}^{I}, y_{i}^{I}\right) Z\left(s_{i}^{I I}, y_{i}^{I I}\right)
$$

$$
\mathbb{V}\left[Z_{Y_{0}, Y_{1}}\right] \approx \frac{1}{M} \sum_{i=1}^{M} Z\left(s_{i}^{I}, y_{i}^{I}\right) Z\left(s_{i}^{I I}, y_{i}^{I}\right)-\frac{1}{M} \sum_{i=1}^{M} Z\left(s_{i}^{I}, y_{i}^{I}\right) Z\left(s_{i}^{I I}, y_{i}^{I I}\right),
$$

where $\left\{s_{i}^{I}\right\}_{i=1}^{M} \subseteq \mathbb{R}^{2}$ and $\left\{s_{i}^{I I}\right\}_{i=1}^{M} \subseteq \mathbb{R}^{2}$ denote two sets of joint realizations of $\left(S_{0}(t), S_{1}(t)\right)$, and $\left\{y_{i}^{I}\right\}_{i=1}^{M} \subseteq \mathbb{R}^{2}$ and $\left\{y_{i}^{I I}\right\}_{i=1}^{M} \subseteq \mathbb{R}^{2}$ denote two sets of joint realizations of $\left(Y_{0}, Y_{1}\right)$, being all of them independent. The notation used here, for instance $Z\left(s_{i}^{I}, y_{i}^{I}\right)$, means evaluating $Z(x, t)$ (given by (11)) at those specific realizations. In Figure 6, we report the estimates of the Sobol indices for $t=10$. We observe that $\mathbb{S}_{Y_{0}, Y_{1}}$ is very close to 1 , therefore indicating that the variability of $Z(x, t)$ is dominated by the initial conditions. Thus we expect Procedure 2 to be superior to Procedure 1 in terms of variance reduction.

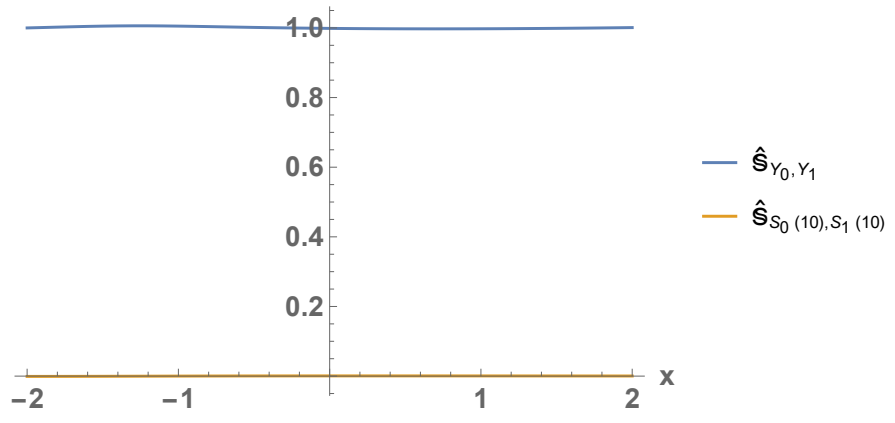

FIG. 6: Estimated Sobol indices of the random inputs for $Z(x, t=10)$. This figure corresponds to Example 3.

In this example, the Gaussian quadrature rule is based on Hermite polynomials. We work at time $t=10$, with 
degree of quadrature $Q=30$. Essentially, the quadrature rule approximation is error-free. In Figure 7 we plot estimates $\hat{\mathbb{V}}[Z(x, 10)]$ and $\hat{\mathbb{V}}\left[\mathbb{E}\left[Z(x, 10) \mid S_{0}(10), S_{1}(10)\right]\right]$. Observe that the magnitude of $\hat{\mathbb{V}}\left[\mathbb{E}\left[Z(x, 10) \mid S_{0}(10), S_{1}(10)\right]\right]$ is smaller than $\hat{\mathbb{V}}[Z(x, 10)]$, due to the law of total variance (18). Anyway, none of the variances becomes large at any point $x$, therefore good estimates of $f_{X(t=10)}(x)$ are obtained with any of the two algorithms.

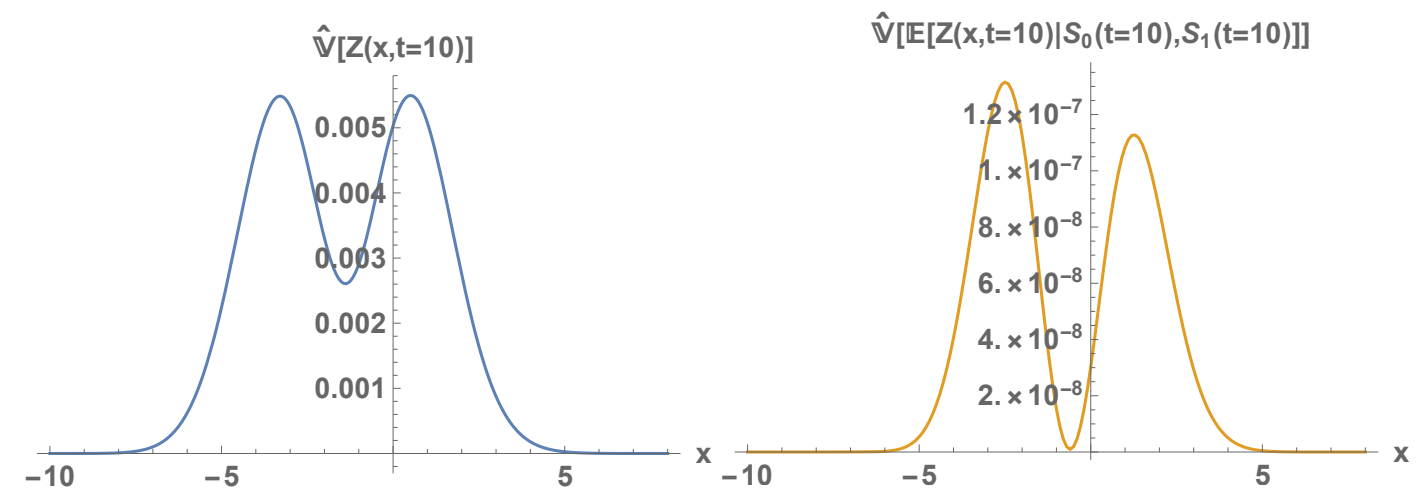

FIG. 7: Estimates of $\hat{\mathbb{V}}\left[\mathbb{E}\left[Z(x, 10) \mid S_{0}(10), S_{1}(10)\right]\right]$ and $\hat{\mathbb{V}}[Z(x, 10)]$. This figure corresponds to Example 3.

In Figure 8 we depict the estimates for $f_{X(t=10)}(x)$ with Procedure 1 (legend "Density MC") and Procedure 2 (legend "Density MCQ"). Very similar smooth estimates are obtained, in fact they overlap, with $Q=30$ and $M=$ 10,000 realizations. We also compare the two estimates with the exact density function (legend "Exact density"). The exact density function is computable using a cubature rule for the double integral (4) (cubature rule constructed using a tensor product approach based on univariate Gauss-Hermite and Gauss-Legendre quadrature rules), because the integrand is smooth with no peaks (see Figure 9), thus posing no difficulties. The second panel of the figure shows the plot in logarithmic scale, to better appreciate the agreement at the tails.

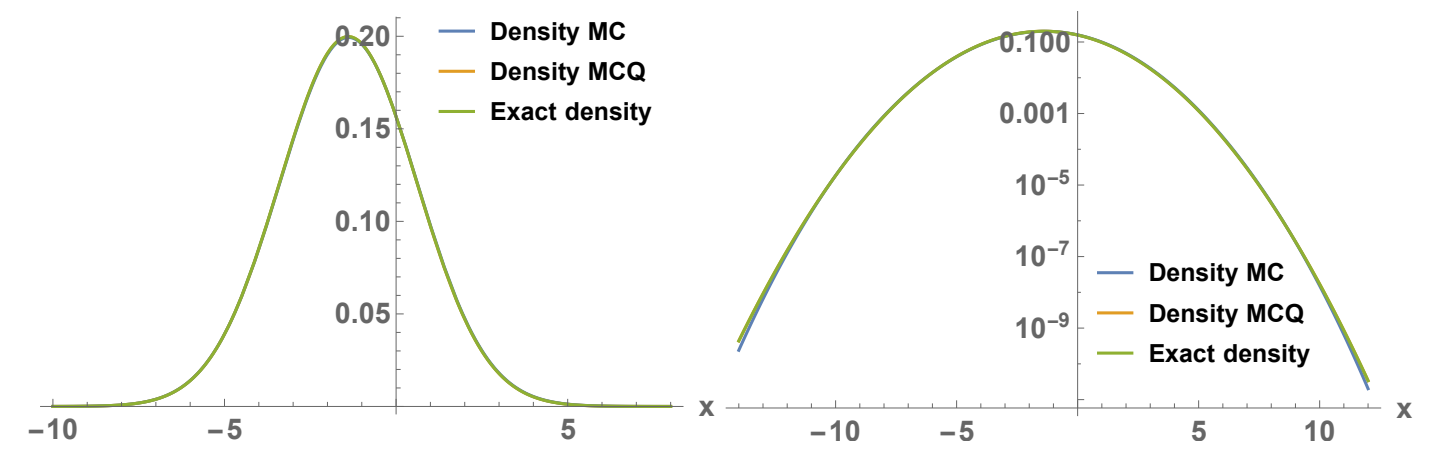

FIG. 8: Estimates of $f_{X(t=10)}(x)$ with MC simulation, MC simulation plus quadrature rule (MCQ), and exact density function. The right panel shows the plot in logarithmic scale. This figure corresponds to Example 3. 

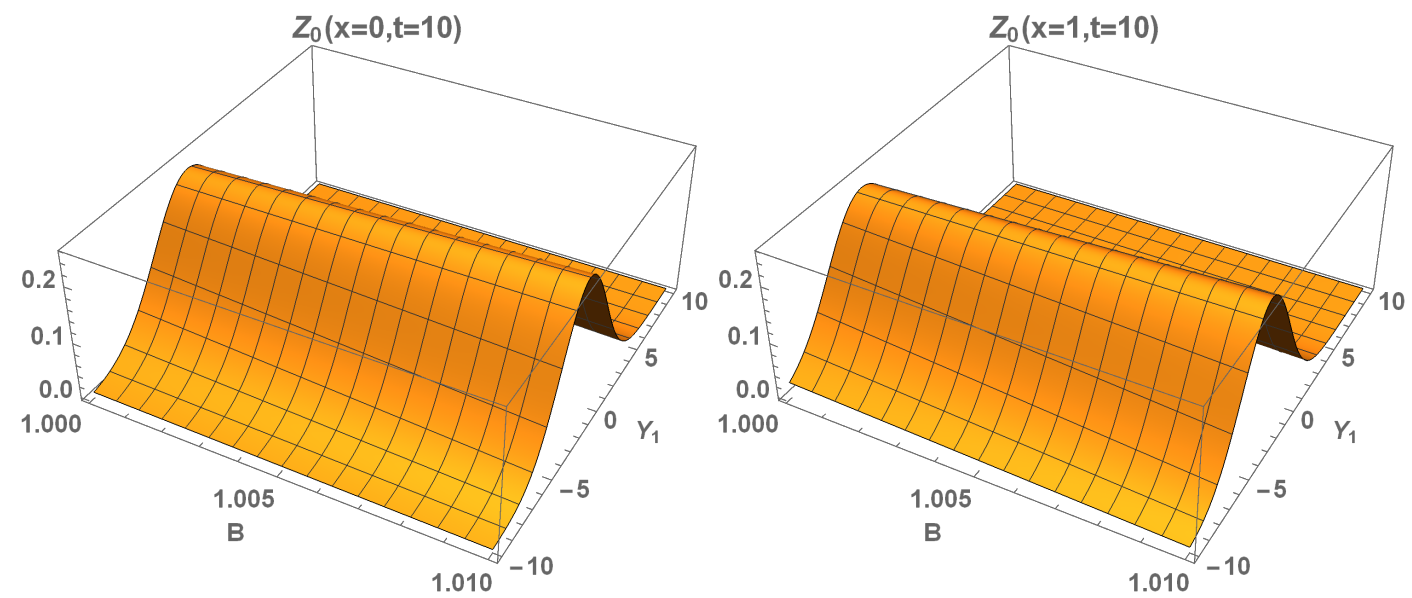

FIG. 9: 3D plots of the integrand $Z_{0}(x, t=10)$ at different points $x$. This figure corresponds to Example 3.

A further analysis is performed by studying the relation between the error and the complexity of Procedure 1 and Procedure 2. In Figure 10 we show plots of mean relative error versus complexity in log-log scale for Procedure 1 (legend "MC") and Procedure 2 (legend "MCQ"), at different points $x$. Here we consider the complexity of Procedure 1 as $M$, while the complexity of Procedure 2 as $M Q$, being $Q=30$ (recall that $M$ denotes the number of realizations). The relative error is defined as the absolute error divided by the value of the exact density function. We run both algorithms up to complexity floor $\left(1.4^{44}\right)$, and we keep the relative errors for complexities floor $\left(1.4^{i}\right)$, $i=14,15, \ldots, 44$ (a geometric progression makes equidistant complexities in log-scale). This gives a discretized realizable path of relative errors. We repeat this demanding process $n=20$ times with parallel computing to obtain an estimate of the mean relative error (so that the analysis performed does not depend upon a specific realizable relative error). A $95 \%$ confidence interval of Gaussian-type for the mean relative error is constructed (this is justified by the central limit theorem), as mean plus/minus 1.96 times the standard error, where the standard error is the standard deviation of the original sample of length $n$ divided by $\sqrt{n}{ }^{*}$. The relative error descends with slope $1 / 2$ approximately, because the error of the MC simulation goes down with $M$ at rate $1 / \sqrt{M}$. The mean relative error of Procedure 2 is shifted down the mean relative error of Procedure 1, due to the variance reduction of the estimators, thus indicating higher efficiency. Similar plots have been obtained for the points $x \in\{-1,3\}$. Indeed, the effect of the initial conditions on the variability of $Z$ is homogeneous in $x$, according to the Sobol indices from Figure 6, therefore

\footnotetext{
*Here $n$ might not be that large to apply the central limit theorem. Other types of confidence intervals may be considered, for instance those constructed via bootstrapping [36] with $b$ samples with replacement from the original sample of length $n$. The percentile confidence interval has as left endpoint the empirical 0.025 percentile, and as right endpoint the empirical 0.975 percentile [36, p. 170], [37, p. 203]. The pivotal confidence interval generated by bootstrapping has left endpoint given by twice the mean minus the empirical 0.975 percentile, and right endpoint given by twice the mean minus the empirical 0.025 percentile [37, p. 194]. Finally, another Gaussian-type confidence interval may consider the mean plus/minus 1.96 times the bootstrap standard deviation of the mean. In all our examples, we checked that these confidence intervals are coinciding, thus showing robustness of our estimates. As $n$ increases, better estimates for the mean are obtained.
} 
a similar performance of the algorithm is expected for the different $x$ 's.
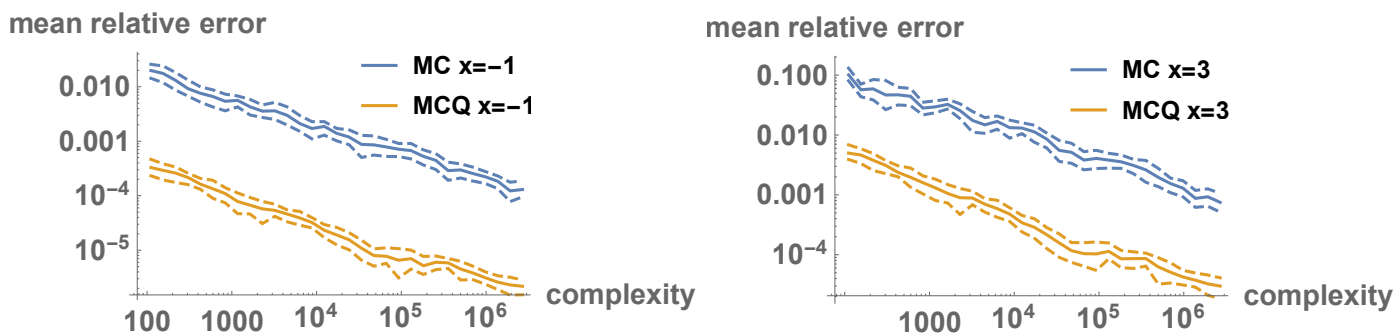

FIG. 10: Plots in log-log scale of mean relative error versus complexity, at $t=10$. The dashed lines represent $95 \%$ confidence intervals. This figure corresponds to Example 3.

Example 4. We consider the same setting as Example 1: (15) with $B \sim \operatorname{Uniform}(1,2.5)$ and $Y_{0}, Y_{1} \sim \operatorname{Normal}(1, \sigma=$ 0.1 ), all of them independent.

In this case, the variability of $Z$ is mainly due to $B$. This is deduced from Figure 11, where we report the Sobol indices (19) and (20) for $t=10$, using the MC estimates (24) and (25). The variance decomposition of $Z$ is dominated by the effect of the fundamental set and the interactions, being the estimated Sobol index corresponding to the initial conditions very close to 0 . Therefore, we do not expect much variance reduction from the quadrature rule.

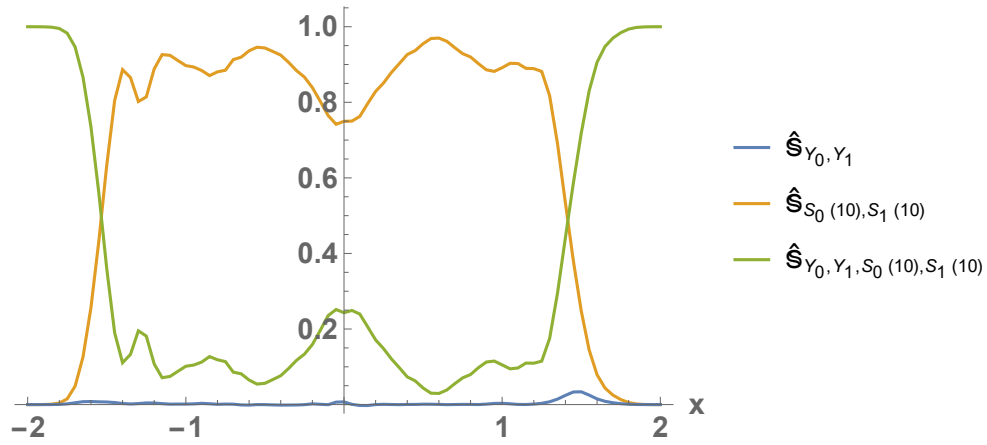

FIG. 11: Estimated Sobol indices of the random inputs for $Z(x, t=10)$. This figure corresponds to Example 4 .

We work at $t=10$, with degree of quadrature $Q=30$ and Hermite polynomials. In Figure 12, left panel, we plot the variances of the statistical estimators from Procedure 1 and Procedure 2. We observe that the variances are greater than in Example 3. Also, in contrast with Example 3, the variance is not much reduced now with the quadrature rule. This was already expected when analyzing the effect of the inputs on the variability of $Z$ using Sobol indices. Figure 12, right panel, depicts the estimated density function $f_{X(t=10)}(x)$ with Procedure 1 (legend "MC") and Procedure 2 (legend "MCQ"), by setting $M=40,000$ realizations and $Q=30$. Both algorithms show similar 
estimates.

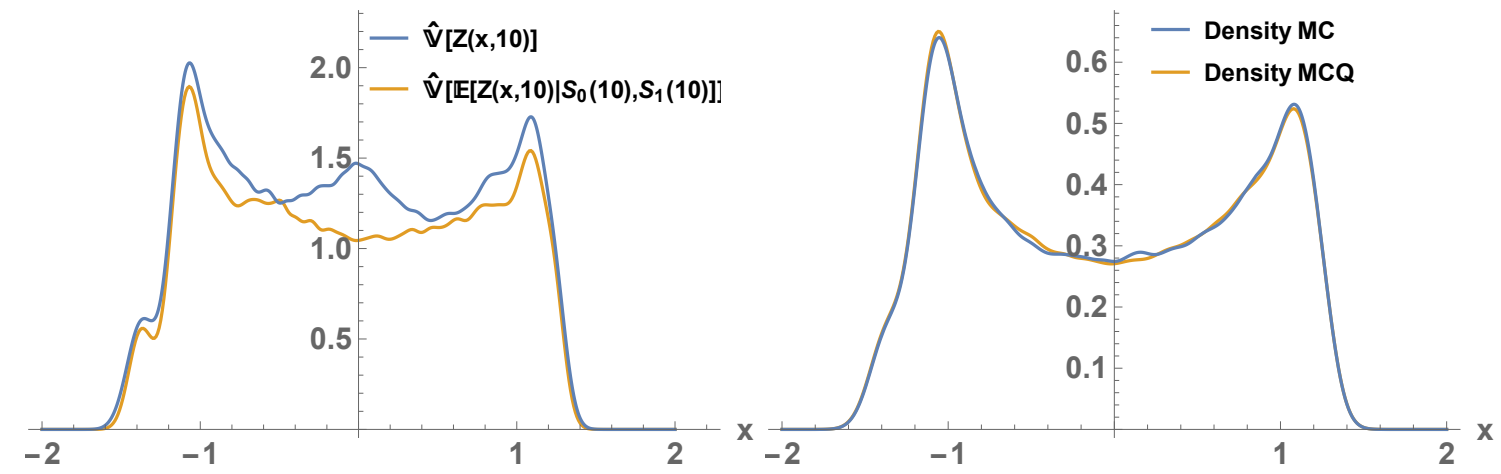

FIG. 12: Left: estimates of $\hat{\mathbb{V}}\left[\mathbb{E}\left[Z(x, 10) \mid S_{0}(10), S_{1}(10)\right]\right]$ and $\hat{\mathbb{V}}[Z(x, 10)]$. Right: estimates of $f_{X(t=10)}(x)$ with MC simulation, and $\mathrm{MC}$ simulation plus quadrature rule (MCQ). This figure corresponds to Example 4.

Figure 13 reports the log-log relationship between the relative error and the complexity at $t=10$, for different points $x$. The setting is analogous to Example 3. We consider the complexities of Procedure 1 and Procedure 2 as $M$ and $M Q$, respectively, where $Q=30$ and $M$ is the number of realizations. The "absolute error" is calculated with respect to the density function obtained from Procedure 1 with 100,000, 000 realizations (this is considered as our "exact density function"). We define the relative error as such absolute error divided by the value of the "exact density function". We run both algorithms up to complexity floor $\left(1.4^{44}\right)$, while keeping the relative errors for complexities floor $\left(1.4^{i}\right), i=14,15, \ldots, 44$. The procedure is repeated $n=20$ times to determine an estimate for the mean relative error. A 95\% Gaussian confidence interval for the mean relative error is constructed. See Example 3 for further details. We observe that the relative errors decay approximately with slope $1 / 2$, due to the rate of convergence proportional to $1 / \sqrt{M}$ of the MC simulation. In this example, the decay rate with slope $1 / 2$ is true for both algorithms as long as the sampling error of the "exact density function" does not become dominant. In the figure, we observe that the estimated relative error of Procedure 1 tends to be constant sometime after $10^{6}$ complexity, as it becomes of similar order of magnitude to the sampling error of the "exact density function". Procedure 1 shows superior to Procedure 2 in this example: in the plots, the depicted relation between the error and the complexity of Procedure 2 is shifted to the right, because of the multiplication by $Q$ of the number of realizations $M$ in the complexity. As the effect of the initial conditions on the variability of $Z$ is small, the potentiality of Procedure 2 is lessened.

In the two Examples 3, 4 presented until now, we studied two cases. When $Y_{0}-Y_{1}$ produce high variability on $\mathbb{V}[Z(x, t)]$, then Procedure 2 clearly wins Procedure 1 in terms of error versus complexity. On the contrary, when the dispersion of $Y_{0}-Y_{1}$ is small compared to $S_{0}(t)$ and $S_{1}(t)$, Procedure 2 does no longer beat Procedure 1. But in 

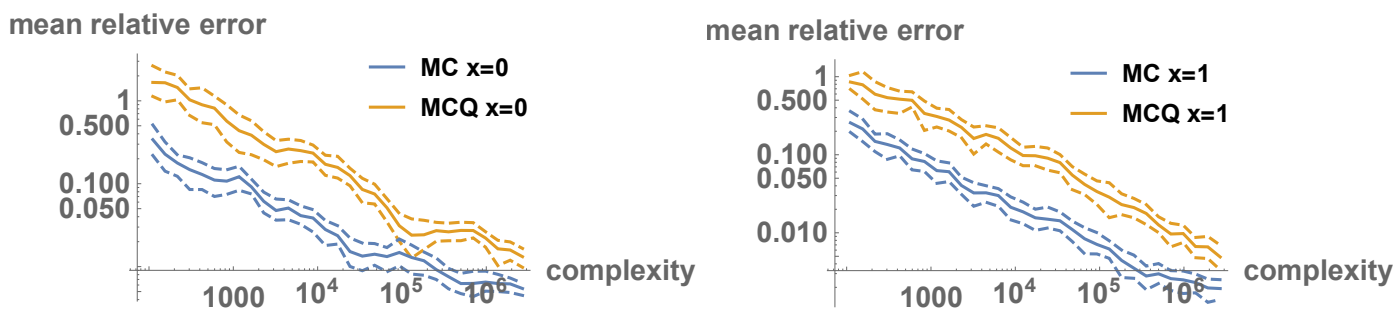

FIG. 13: Plots in log-log scale of mean relative error versus complexity, at $t=10$. The dashed lines represent $95 \%$ confidence intervals. This figure corresponds to Example 4.

general, when the cost of obtaining realizations of $S_{0}(t)$ and $S_{1}(t)$ is larger (because we are working with truncated power series in $N$, for instance), the cost due to the $\mathrm{MC}$ procedure alone is comparable with the whole complexity. In such a case, Procedure 1 never defeats Procedure 2 by a big difference. This is the case studied in the following Example 5.

Example 5. Consider (16) with inputs $A_{0}=4, A_{1} \sim \operatorname{Uniform}(0,1), B_{0} \sim \operatorname{Triangular}(-1,0), B_{1} \sim \operatorname{Bernoulli}(0.35)$ and $Y_{0}, Y_{1} \sim \operatorname{Normal}(1, \sigma=0.1)$, all of them independent. The random coefficients $A_{0}, A_{1}, B_{0}$ and $B_{1}$ are bounded and the initial conditions $Y_{0}$ and $Y_{1}$ have finite absolute moments, therefore there is an analytic solution $X(t)$ in the $\mathrm{L}^{p}(\Omega)$ sense, $1 \leq p<\infty$.

In this example, the fundamental set cannot be written with simple closed-form formulas. One needs to perform a dimensionality reduction of the problem by truncating the random power series at certain order $N$. We work at time $t=1.5$ with order of truncation $N=15$. The goal is to compare the approximations to the density $f_{X^{N=15}(t=1.5)}(x)$ offered by Procedure 1 and Procedure 2.

In Figure 14 we show the Sobol indices (19) and (20) for $t=1.5$, computed with the MC estimates (24) and (25). The Sobol indices demonstrate that the effect of $Y_{0}-Y_{1}$ on the variability of $Z^{N=15}(x, t=1.5)$ is very small.

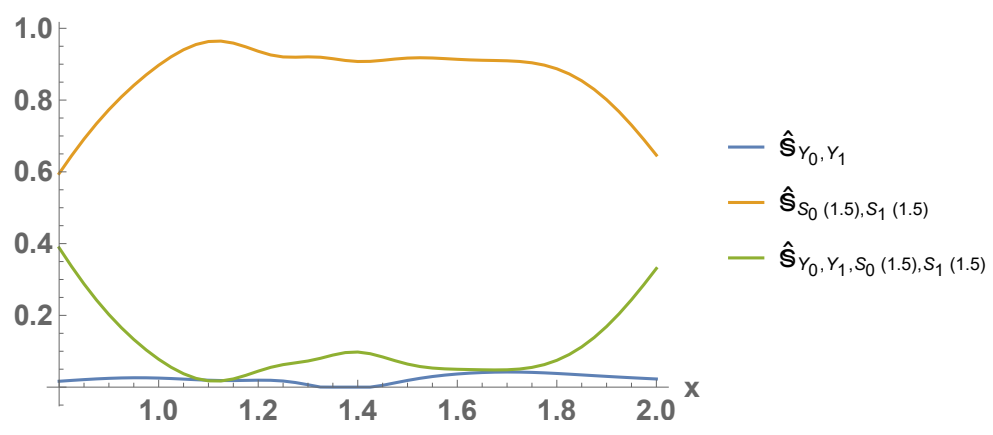

FIG. 14: Estimated Sobol indices of the random inputs for $Z^{N=15}(x, t=1.5)$. This figure corresponds to Example 5 . 
Hence, the variance reduction due to the quadrature rule is not very significant. This is confirmed by Figure 15, left panel, where we depict the variances of the statistical estimators. We use quadrature degree $Q=30$, so that the expectation with respect to $Y_{0}-Y_{1}$ is practically error-free. In the right plot, we estimate the density function $f_{X^{N=15}(t=1.5)}(x)$ using Procedure 1 and Procedure 2, in logarithmic scale. We generated $M=10,000$ realizations.
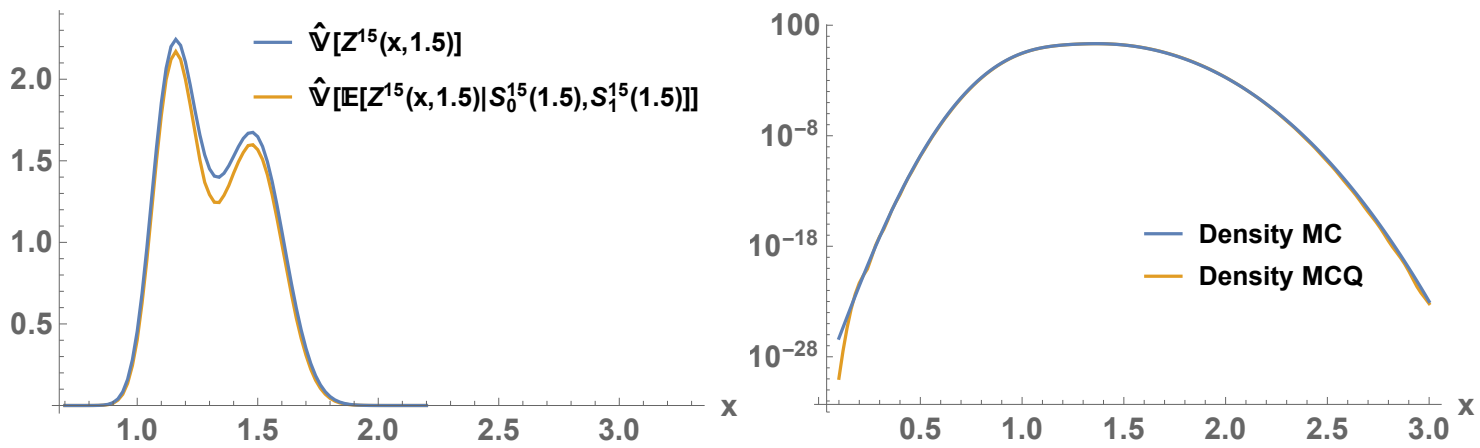

FIG. 15: Left: estimates of $\hat{\mathbb{V}}\left[\mathbb{E}\left[Z^{15}(x, 1.5) \mid S_{0}(1.5), S_{1}(1.5)\right]\right]$ and $\hat{\mathbb{V}}\left[Z^{15}(x, 1.5)\right]$. Right: estimates of $f_{X^{15}(1.5)}(x)$ (the legend MCQ refers to MC plus quadrature rule), in logarithmic scale. This figure corresponds to Example 5.

An analogous analysis to Example 4 of relative error versus complexity is carried out here. The complexity of Procedure 1 is $M N$, whereas the complexity of Procedure 2 is $M(N+Q)$. Recall that $M$ is the number of realizations, $N$ is the truncation order for the power series, and $Q$ is the quadrature degree. We work at the point $x=1.4$, which belongs to the density domain (any other point is equally valid). We consider as our "exact density function" the output of Procedure 1 with $M=10,000,000$ realizations, i.e., complexity $M N=150,000,000$. We run both algorithms for complexities floor $\left(1.4^{37}\right)$, and keep the relative errors for complexities floor $\left(1.4^{i}\right), i=14,15, \ldots, 37$. The procedure is repeated $n=20$ times (in parallel) to obtain the mean relative error. A 95\% Gaussian confidence interval for the mean relative error has been constructed. Procedure 2 is run with $Q=10$ and $Q=30$ degrees of quadrature. Figure 16 shows the results. Notice that, although $Y_{0}-Y_{1}$ have small dispersion compared with the fundamental set, both Algorithms show similar performances. Indeed, as each realization of the truncated fundamental set requires now more expense ( $\operatorname{cost} N$ ), Procedure 1 cannot defeat Procedure 2 by a big difference. The relative errors decrease with slope $1 / 2$, but for $Q=10$ we perceive constant error for complexities greater than $10^{5}$. This is because the bias error caused by the quadrature rule of degree $Q=10$ has been attained, in contrast to the case $Q=30$. This reflects the importance of selecting a sufficiently large quadrature degree, especially when $f_{Y_{0}}$ and $f_{Y_{1}}$ are peaked by small dispersion of $Y_{0}$ and $Y_{1}$. 


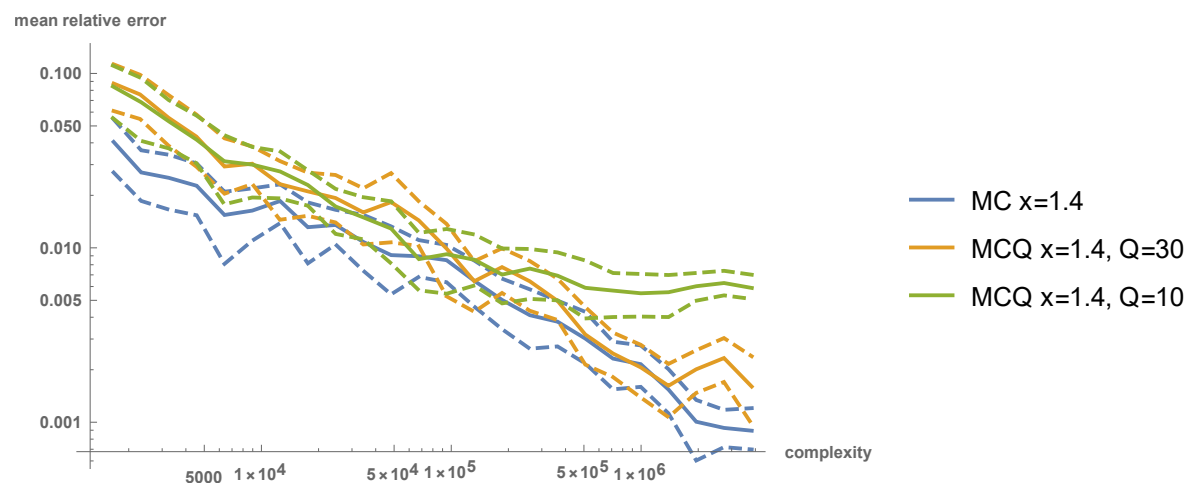

FIG. 16: Plots in log-log scale of mean relative error versus complexity, at $t=1.5$ and $N=15$. The dashed lines represent $95 \%$ confidence intervals. This figure corresponds to Example 5.

Case 1 When the dispersion of $Y_{0}-Y_{1}$ is large compared with $S_{0}(t)$ and $S_{1}(t)$ (this is checked with Sobol indices), then Procedure 2 should be used. See Example 3.

Case 2 When the variability of $Y_{0}-Y_{1}$ is small compared with $S_{0}(t)$ and $S_{1}(t)$ (Sobol indices), and each realization of $S_{0}(t)$ and $S_{1}(t)$ takes certain computational burden (for instance, because we are dealing with truncated power series), then Procedure 1 and Procedure 2 are similar in terms of error versus complexity. See Example 5.

Case 3 Finally, if the variability of $Y_{0}-Y_{1}$ is small compared with $S_{0}(t)$ and $S_{1}(t)$ (Sobol indices), and each realization of $S_{0}(t)$ and $S_{1}(t)$ is immediately computed (when $S_{0}(t)$ and $S_{1}(t)$ are known with simple closed-form expressions), then Procedure 1 beats Procedure 2. See Example 4.

In any of the three cases, $Q$ should be taken large enough to avoid a quadrature error greater than the sampling error.

\section{MULTILEVEL MC SIMULATION}

The idea of multilevel MC $[27,28]$ is to speed up the convergence of the MC simulation by decomposing the target expectation into the sum of expectations of increments (through a telescopic sum identity), whose variances decay rapidly. In our case, we consider the following increments:

$$
\Delta Z_{0}^{l}(x, t)=Z_{0}^{l+1}(x, t)-Z_{0}^{l}(x, t), \quad \Delta Z_{1}^{l}(x, t)=Z_{1}^{l+1}(x, t)-Z_{1}^{l}(x, t),
$$


for each level $l \geq 0$, where each term was defined in (10). If we start from the level $l_{0} \geq 0$, these increments yield the following telescopic sum identities, for $i \in\{1,2\}$ :

$$
f_{X(t)}(x)=\mathbb{E}\left[Z_{i}^{l_{0}}(x, t)\right]+\sum_{l=l_{0}}^{\infty} \mathbb{E}\left[\Delta Z_{i}^{l}(x, t)\right] \approx f_{X^{N}(t)}(x)=\mathbb{E}\left[Z_{i}^{l_{0}}(x, t)\right]+\sum_{l=l_{0}}^{N-1} \mathbb{E}\left[\Delta Z_{i}^{l}(x, t)\right]
$$

Each one of these expectations is approximated with MC simulation. The rate of convergence of the MC simulation depends on the number of realizations and $\mathbb{V}\left[Z_{0}^{l_{0}}(x, t)\right], \mathbb{V}\left[\Delta Z_{0}^{l}(x, t)\right]$, and $\mathbb{V}\left[Z_{1}^{l_{0}}(x, t)\right], \mathbb{V}\left[\Delta Z_{1}^{l}(x, t)\right]$. If $Z_{0}^{l}(x, t)$ or $Z_{1}^{l}(x, t)$ tend to $Z^{l}(x, t)$ in the mean square sense, then the variances of the increments, $\mathbb{V}\left[\Delta Z_{0}^{l}(x, t)\right], \mathbb{V}\left[\Delta Z_{1}^{l}(x, t)\right]$, converge to 0 , which makes the convergence of the MC procedure faster. The first level $l_{0}$ is usually chosen satisfying that, for $l \geq l_{0}$, the variances of the increments per level $l$ are decaying monotonically and rapidly.

However, as we are selecting either $f_{Y_{0}}$ or $f_{Y_{1}}$ (as in [25]), this may lead to numerical instabilities due to small denominators and large or infinite variance, which causes noisy features and slows down the convergence of the MC procedure. This may hide the potential of multilevel MC.

To sort out this issue, we path-wise select $\Delta Z_{0}^{l}(x, t)$ or $\Delta Z_{1}^{l}(x, t)$, by discarding the increment with the lowest denominator. The minimum among the denominators is considered as a time-dependent random variable

$$
V^{l}(t)=\min \left\{\left|S_{0}^{l+1}(t)\right|,\left|S_{0}^{l}(t)\right|,\left|S_{1}^{l+1}(t)\right|,\left|S_{1}^{l}(t)\right|\right\} .
$$

We pick the index with no smallest denominator through the random variable

$$
U^{l}(t)=\left\{1-i: \text { the minimum of } V^{l}(t) \text { is attained at } i \in\{0,1\}\right\} .
$$

This random variable gives rise to two events, $H_{0}^{l}(t)=\left\{U^{l}(t)=0\right\}$ and $H_{1}^{l}(t)=\left\{U^{l}(t)=1\right\}$. Let $Z_{\Delta}^{l}(x, t)=$ $\Delta Z_{0}^{l}(x, t) 1_{H_{0}^{l}(t)}+\Delta Z_{1}^{l}(x, t) 1_{H_{1}^{l}(t)}$. This random process models the selection of $\Delta Z_{0}^{l}(x, t)$ and $\Delta Z_{1}^{l}(x, t)$, according to whether their expressions do not have the smallest denominator.

\section{Proposition 4. We have the following sum identities for $f_{X(t)}(x)$ and $f_{X^{N}(t)}(x)$ :}

$$
f_{X(t)}(x)=\mathbb{E}\left[Z^{l_{0}}(x, t)\right]+\sum_{l=l_{0}}^{\infty} \mathbb{E}\left[Z_{\Delta}^{l}(x, t)\right], f_{X^{N}(t)}(x)=\mathbb{E}\left[Z^{l_{0}}(x, t)\right]+\sum_{l=l_{0}}^{N-1} \mathbb{E}\left[Z_{\Delta}^{l}(x, t)\right]
$$

With the objective of approximating the target density function $f_{X(t)}(x)$ pointwise, we truncate the telescopic sum identity at increment $N-1$, which causes the bias error $\theta_{N}(x, t)=f_{X(t)}(x)-f_{X^{N}(t)}(x): f_{X(t)}(x) \approx$ 
$f_{X^{N}(t)}(x)=\mathbb{E}\left[Z^{l_{0}}(x, t)\right]+\sum_{l=l_{0}}^{N-1} \mathbb{E}\left[Z_{\Delta}^{l}(x, t)\right]$. The expectations $\mathbb{E}\left[Z^{l_{0}}(x, t)\right], \mathbb{E}\left[Z_{\Delta}^{l}(x, t)\right], l=l_{0}, \ldots, N-1$, are

3

4

computed independently with MC simulation, whose rate of convergence depends on the finite number of realizations and on $\mathbb{V}\left[Z^{l_{0}}(x, t)\right], \mathbb{V}\left[Z_{\Delta}^{l}(x, t)\right], l=l_{0}, \ldots, N-1$.

We have $\left\|Z_{\Delta}^{l}(x, t)\right\|_{2} \leq\left\|\Delta Z_{0}^{l}(x, t)\right\|_{2}+\left\|\Delta Z_{1}^{l}(x, t)\right\|_{2}$. Thus, if both $\Delta Z_{0}^{l}(x, t)$ and $\Delta Z_{1}^{l}(x, t)$ have finite variances, then so does $Z_{\Delta}^{l}(x, t)$. On the contrary, if $\Delta Z_{0}^{l}(x, t)$ or $\Delta Z_{1}^{l}(x, t)$ have infinite variances, then $Z_{\Delta}^{l}(x, t)$ may have finite variance, instead. In any of these cases, we cannot get worse in terms of infinite variance.

We hope to have the variances $\mathbb{V}\left[Z^{l_{0}}(x, t)\right]$ and $\mathbb{V}\left[Z_{\Delta}^{l}(x, t)\right], l=l_{0}, \ldots, N-1$, finite, to exploit the decay of $\mathbb{V}\left[Z_{\Delta}^{l}(x, t)\right]$ to 0 with $l$. The first level $l_{0}$ is chosen such that $\mathbb{V}\left[Z_{\Delta}^{l}(x, t)\right]$ is decreasing with $l \geq l_{0}$, monotonically and fast. The best case is when $\log \mathbb{V}\left[Z_{\Delta}^{l}(x, t)\right]$ is decreasing linearly with $l \geq l_{0}$, as exponential decay holds for the variances of the increments.

Let $M=\left(N_{l_{0}} ; M_{l_{0}}, \ldots, M_{N-1}\right)$ be the number of simulations performed: $N_{l_{0}}$ for $Z^{l_{0}}(x, t), M_{l}$ for $Z_{\Delta}^{l}(x, t)$, $l=l_{0}, \ldots, N-1$. In principle, the number of simulations may depend on $(x, t)$, although we drop this possible dependence along this discussion.

Let $f_{X}^{N, M}(x, t)$ be the final approximation to $f_{X(t)}(x)$ :

$$
f_{X}^{N, M}(x, t)=\frac{1}{N_{l_{0}}} \sum_{i=1}^{N_{l_{0}}} Z^{l_{0}}\left(x, t, \omega_{i, l_{0}}\right)+\sum_{l=l_{0}}^{N-1} \frac{1}{M_{l}} \sum_{i=1}^{M_{l}} Z_{\Delta}^{l}\left(x, t, \omega_{i, l, \Delta}\right)
$$

where $\omega$ with the appropriate subindices denote outcomes. The mean square error in the approximation to $f_{X^{N}(t)}(x)=$ $\mathbb{E}\left[f_{X}^{N, M}(x, t)\right]$ is given by the mean square norm of $\mathcal{E}_{N, M}(x, t)=f_{X^{N}(t)}(x)-f_{X}^{N, M}(x, t)$, which is

$$
\left\|f_{X^{N}(t)}(x)-f_{X}^{N, M}(x, t)\right\|_{2}^{2}=\mathbb{V}\left[\mathcal{E}_{N, M}(x, t)\right]=\mathbb{V}\left[f_{X}^{N, M}(x, t)\right]=\frac{\mathbb{V}\left[Z^{l_{0}}(x, t)\right]}{N_{l_{0}}}+\sum_{l=l_{0}}^{N-1} \frac{\mathbb{V}\left[Z_{\Delta}^{l}(x, t)\right]}{M_{l}}
$$

Notice that the first equality holds because $\mathbb{E}\left[\mathcal{E}_{N, M}(x, t)\right]=0$, so the squared norm is equal to the variance. The second equality uses the fact that $f_{X^{N}(t)}(x)$ is not random. Finally, the third equality is deduced by applying the variance in (29). The total cost is

$$
W_{N, M}=N_{l_{0}} c_{l_{0}}+\sum_{l=l_{0}}^{N-1} M_{l} C_{l}
$$

where $c_{l_{0}}$ denotes the number of operations per realization of $Z^{l_{0}}(x, t)$, and $C_{l}$ denotes the number of operations per realization of $Z_{\Delta}^{l}(x, t), l=l_{0}, \ldots, N-1$. In our case, we consider $c_{l}=C_{l}=l^{2}$ when dealing with $A(t)$ and $B(t)$ as random infinite expansions, and $c_{l}=C_{l}=l$ when $A(t)$ and $B(t)$ are random polynomials. 
Proposition 5. Given a fixed variance (30) $\epsilon_{\text {sampling, }}^{2}$ the cost (31) is minimized by selecting

$$
N_{l_{0}}(x, t)=\epsilon_{\text {sampling }}^{-2}\left(\sqrt{\mathbb{V}\left[Z^{l_{0}}(x, t)\right] c_{l_{0}}}+\sum_{l=l_{0}}^{N-1} \sqrt{\mathbb{V}\left[Z_{\Delta}^{l}(x, t)\right] C_{l}}\right) \sqrt{\frac{\mathbb{V}\left[Z^{l_{0}}(x, t)\right]}{c_{l_{0}}}},
$$

$$
M_{l}(x, t)=\epsilon_{\text {sampling }}^{-2}\left(\sqrt{\mathbb{V}\left[Z^{l_{0}}(x, t)\right] c_{l_{0}}}+\sum_{l=l_{0}}^{N-1} \sqrt{\mathbb{V}\left[Z_{\Delta}^{l}(x, t)\right] C_{l}}\right) \sqrt{\frac{\mathbb{V}\left[Z_{\Delta}^{l}(x, t)\right]}{C_{l}}},
$$

3 for $l=l_{0}, \ldots, N-1$. In such a case, the total cost (31) is given by

$$
W_{N, M}=\epsilon_{\text {sampling }}^{-2}\left(\sqrt{\mathbb{V}\left[Z^{l_{0}}(x, t)\right] c_{l_{0}}}+\sum_{l=l_{0}}^{N-1} \sqrt{\mathbb{V}\left[Z_{\Delta}^{l}(x, t)\right] C_{l}}\right)^{2} .
$$

This proposition is a simple application of Lagrange multipliers.

For a truncation order $N$, and for a sampling error variance $\epsilon_{\text {sampling }}^{2}$ by running $\epsilon_{\text {sampling }}^{-2} \mathbb{V}\left[Z^{N}(x, t)\right]$ simulations, the cost of Procedure 1 is $\epsilon_{\text {sampling }}^{-2} \mathbb{V}\left[Z^{N}(x, t)\right] c_{N}$. For fixed $N$, both approaches (standard MC in Procedure 1 and multilevel MC) yield a cost $\mathcal{O}\left(\epsilon_{\text {sampling }}^{-2}\right)$. However, there is a significant difference when $N$ grows. The complexity $c_{N}=N$ (if $A(t)$ and $B(t)$ are random polynomials) or $c_{N}=N^{2}$ (if $A(t)$ and $B(t)$ are infinite random series) per realization of $Z^{N}(x, t)$ grows to infinity. Then the cost of Procedure 1 tends to infinity as $N \rightarrow \infty$, linearly (if $c_{N}=$ $N$ ) or quadratically (if $c_{N}=N^{2}$ ). If we choose $N=\mathcal{O}\left(\log \epsilon_{\text {sampling }}^{-1}\right.$ ) to ensure a bias error less than $\epsilon_{\text {sampling }}$ (under exponential convergence of $f_{X^{N}(t)}(x)$ with $\left.N\right)$, then the complexity of Procedure 1 is $\mathcal{O}\left(\epsilon_{\text {sampling }}^{-2} \log ^{2} \epsilon_{\text {sampling }}\right)$, when $c_{N}=N^{2}$, and $\mathcal{O}\left(\epsilon_{\text {sampling }}^{-2} \log \epsilon_{\text {sampling }}^{-1}\right)$, when $c_{N}=N$. However, in the multilevel approach the cost (34) remains $\mathcal{O}\left(\epsilon_{\text {sampling }}^{-2}\right)$ despite having $N \rightarrow \infty$, as $\sqrt{\mathbb{V}\left[Z^{l_{0}}(x, t)\right] c_{l_{0}}}+\sum_{l=l_{0}}^{\infty} \sqrt{\mathbb{V}\left[Z_{\Delta}^{l}(x, t)\right] C_{l}}<\infty$ in general. For instance, when $\mathbb{V}\left[Z_{\Delta}^{l}(x, t)\right]$ decreases exponentially with $l$. Hence, from this theoretical analysis, multilevel MC is superior to standard MC, at least when $N$ grows.

In practice, multilevel $\mathrm{MC}$ is more efficient than standard $\mathrm{MC}$ by choosing the initial level $l_{0}$ and truncation $N>l_{0}$ appropriately, which can be done heuristically as explained later.

In numerical applications, the main computational burden of multilevel MC is due to the number of samples $N_{l_{0}}$ of $Z^{l_{0}}(x, t)$. For the last levels near $N$, which are the most complex ones, we run only few samples. In general, from a certain level $l$, only 1 realization of $Z_{\Delta}^{l}(x, t)$ is required. From a certain order of truncation $N$, the complexity of multilevel MC will be constant; whereas the complexity of the standard MC strategy will be growing up to infinity with $N$, linearly or quadratically. In our setting, multilevel MC turns out to be useful when high accuracy is required.

Given $l_{0}, N>l_{0}$ and $\epsilon_{\text {sampling }}$, the cost of multilevel MC is approximately $\epsilon_{\text {sampling }}^{-2} \mathbb{V}\left[Z^{l_{0}}(x, t)\right] c_{l_{0}}$, because the 
sequence $\mathbb{V}\left[Z_{\Delta}^{l}(x, t)\right] C_{l}$ decreases rapidly with $l$. The expense of standard $\mathrm{MC}$ is $\epsilon_{\text {sampling }}^{-2} \mathbb{V}\left[Z^{N}(x, t)\right] c_{N}$. Assuming that $\mathbb{V}\left[Z^{l_{0}}(x, t)\right] \approx \mathbb{V}\left[Z^{N}(x, t)\right]$, which is quite realistic if $l_{0}$ and $N>l_{0}$ are not too small, the cost of multilevel MC is reduced by factor $c_{l_{0}} / c_{N}$, approximately. The largest reduction is thus obtained for problems (1) with infinite series inputs, since in such a case $c_{N}$ grows quadratically with $N$.

The complete multilevel MC approach is presented in what follows. Fixed the time $t$ and the maximum level $N$, the whole procedure is divided into different parts:

\section{Procedure 3.}

Part 1 We determine $\mathbb{V}\left[Z_{\Delta}^{l}(x, t)\right]$ for $0 \leq l \leq N-1$. They are estimated à la $M C$, as Procedure 1 , but not using an excessive amount $m_{\mathbb{V}}$ of realizations. The main loop over the levels l can be carried out in parallel.

Part 2 We estimate $\mathbb{V}\left[Z^{l}(x, t)\right]$ for $1 \leq l \leq N$ (with parallel computing). Then we set the ratios between the complexities of Procedure 1,

$$
W_{N}^{M C}(t)=\epsilon_{\text {sampling }}^{-2} c_{N} \max _{x} \mathbb{V}\left[Z^{N}(x, t)\right]
$$

and of the multilevel MC strategy with initial level $l_{1}=1, \ldots, N-1$,

$$
W_{l_{1}, N}^{\text {multi }}(t)=\epsilon_{\text {sampling }}^{-2} \max _{x}\left(\sqrt{\mathbb{V}\left[Z^{l_{1}}(x, t)\right] c_{l_{1}}}+\sum_{l=l_{1}}^{N-1} \sqrt{\mathbb{V}\left[Z_{\Delta}^{l}(x, t)\right] C_{l}}\right)^{2} .
$$

These estimates are done via standard MC simulation with not too many realizations $m_{\mathbb{V}}$. The ratio of the complexities is given by

$$
R_{l_{1}, N}(t)=\frac{W_{N}^{M C}(t)}{W_{l_{1}, N}^{\text {multi }}(t)}=\frac{\max _{x} \mathbb{V}\left[Z^{N}(x, t)\right] c_{N}}{\max _{x}\left(\sqrt{\mathbb{V}\left[Z^{l_{1}}(x, t)\right] c_{l_{1}}}+\sum_{l=l_{1}}^{N-1} \sqrt{\mathbb{V}\left[Z_{\Delta}^{l}(x, t)\right] C_{l}}\right)^{2}} .
$$

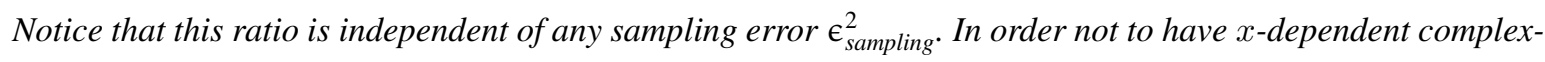
ities, we take the maximum on $x$ lying in the density domain of interest. If $R_{l_{1}, N}(t)>1$, then multilevel MC starting from level $l_{1}$ is more efficient than standard $M C$.

We define the first level $l_{0}=l_{0}(N, t)$ as the maximizer of

$$
\max _{l_{1}: 1 \leq l_{1}<N} R_{l_{1}, N}(t)>1
$$

This way we are achieving the maximum optimality of multilevel $M C$. In general, the variances $\mathbb{V}\left[Z_{\Delta}^{l}(x, t)\right]$ 
start to decrease monotonically and fast with $l$ before the maximizer $l_{0}$.

Part 3 Finally, we perform the whole multilevel MC procedure. Given a squared norm of the sampling error $\epsilon_{\text {sampling }}^{2}$ (see (30)), and given the first level $l_{0}$ obtained in Part 2, we obtain the optimal $N_{l_{0}}(x, t)$ and $M_{l}(x, t)$ for $l=l_{0}, \ldots, N-1$, using (32) and (33) from the constrained minimization problem. We set $N_{l_{0}}(t)$ and $M_{l}(t)$ as the ceiling of the maximum on $x$ lying in the density domain of interest. Thus, the number of simulations is an integer independent of $x$.

Once the number of simulations is fixed, we estimate $\mathbb{E}\left[Z^{l_{0}}(x, t)\right]$ and $\mathbb{E}\left[Z_{\Delta}^{l}(x, t)\right], l=l_{0}, \ldots, N-1$, using standard MC simulation. The output discretized function $f_{X}^{N, M}(x, t)$ is (29), which estimates $f_{X^{N}(t)}(x)$ with

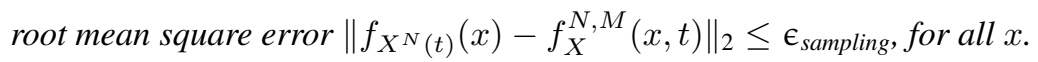

In general, the squared norm of the sampling error $\epsilon_{\text {sampling }}^{2}$ and the order of truncation $N$ are chosen as follows. At the beginning, one can estimate the bias error of the density approximation: given a very large order of truncation $N_{\infty} \gg 1$, and taking into account that $Z^{N_{\infty}}(x, t) \approx Z(x, t)$, the bias error at order $1 \leq N \ll N_{\infty}, \mid f_{X(t)}(x)-$ $f_{X^{N}(t)}(x) \mid$, is estimated as

$$
\left|\widehat{\mathbb{E}}\left[Z^{N_{\infty}}(x, t)-Z^{N}(x, t)\right]\right|
$$

The expectation is estimated using standard MC simulation. Suppose we fix a target global error $\delta$. We split the target

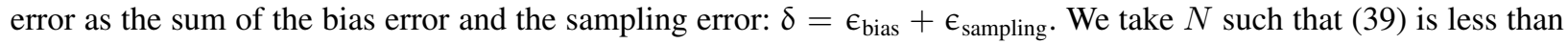
$\epsilon_{\text {bias }}$ for all $x$. Then we apply the multilevel MC procedure with squared norm of the sampling error $\epsilon_{\text {sampling }}^{2}$ Keep in mind that the error $\delta$ cannot be as small as we want, since the sampling error $\epsilon_{\text {sampling }}$ must be achievable.

To apply the multilevel MC procedure, we need $N$ such that the maximum (38) is greater than 1 . This holds for large $N$ for sure, according to the theoretical discussion. Thus, in practice, we can choose $\epsilon_{\text {bias }}$ small to make $N$ large, and then run the multilevel MC algorithm with $\epsilon_{\text {sampling }}=\delta-\epsilon_{\text {bias }}$. In general, the decomposition of $\delta$ into $\epsilon_{\text {bias }}$ and $\epsilon_{\text {sampling }}$ is made heuristically, depending on the size of $N$ for which (38) is greater than 1.

We showcase the multilevel MC method in numerical experiments. The first example deals with (1) having infinite series expansions in the input data; we observe that (38) is greater than 1 for $N \geq 1$, therefore multilevel MC is always superior to standard MC. In the second example, (38) is greater than 1 only for large $N$, so to take advantage of multilevel MC the error $\delta$ is chosen small, so that $N$ is large enough.

These examples highlight what we believe is a general fact. Given $N$, multilevel MC outperforms crude MC simulation when $R_{l_{0}, N}(t)>1$ (see (37) and (38)). But for a certain $N$, it may be possible that $R_{l_{1}, N}(t) \leq 1$ for $1 \leq l_{1}<N$ (this is the case of Example 7 for $N<36$ ). For such an $N$ and the corresponding bias error, MC is more 
efficient than the multilevel approach. When $A(t)$ and $B(t)$ are given by infinite random terms (Example 6), then $c_{l}$, defined as the cost of one realization of $Z^{l}(x, t)$, grows as $l^{2}$, so expression (38) is more inclined to be greater than 1 for small $N$ and higher tolerances (because the numerator of (37) grows quadratically). But when $A(t)$ and $B(t)$ are random polynomials (Example 7), then $c_{l}=l$, so a larger $N$ and lower tolerances are required to ensure that (38) is greater than 1 (assuming that computational resources are available to achieve such an $N$ ).

Example 6. Consider (1) with $t_{0}=0, Y_{0}, Y_{1} \sim \operatorname{Normal}(2,3), A(t)=\sum_{n=0}^{\infty} \frac{U_{n}}{n !} t^{n}$ and $B(t)=t$, where $U_{0}, U_{1}, \ldots$ are independent random variables with distribution $\operatorname{Beta}(11,15)$. The series defining $A(t)$ converges in $\mathrm{L}^{\infty}(\Omega)$ for all $t \in \mathbb{R}$. There is a unique analytic stochastic process $X(t)$ that solves (1) in the $\mathrm{L}^{p}(\Omega)$-sense, $1 \leq p<\infty$.

We estimate $\mathbb{V}\left[Z_{\Delta}^{l}(x, t)\right]$ for $N \leq 20$, using $m_{\mathbb{V}}=10,000$ realizations. With costs $c_{l}=C_{l}=l^{2}$, we estimate the ratios of complexities (37) for $l_{1} \in[1,19], N \in\left[l_{1}+1,20\right]$. In Figure 17 we present the ratios. Observe that they are greater than 1, therefore indicating higher efficiency of multilevel MC compared to crude MC for any level of tolerance. For each $N$, we keep the level $l_{0}$ maximizing the ratio, see (38). For $N \geq 7$, the same maximizer $l_{0}=6$ is obtained.

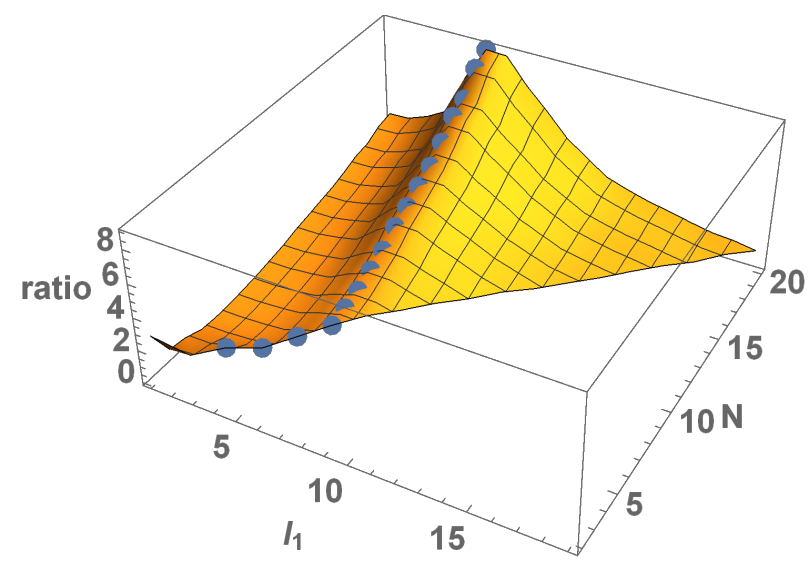

FIG. 17: Ratios of complexities between standard MC and multilevel MC (see (37)). The points indicate the maximum ratio with $l_{1}$ per truncation $N$. This figure corresponds to Example 6.

Figure 18 reports the ratios for $N>l_{0}=6$. The ratios grow roughly quadratically with $N$, with growth constant $1 / l_{0}^{2}$. This is because the complexity of standard MC increases quadratically with $N$, while the cost of multilevel MC becomes constant from a certain level $N$. Small oscillations may be observed because of the finite number of simulations $m_{\mathbb{V}}$ used to estimate the variances.

Once we have the parameters of the multilevel MC strategy, we aim at approximating pointwise the probability density function of $X(t)$ at $t=1.5, f_{X(t=1.5)}(x)$. Figure 19 reports in log-scale the estimated bias errors (39) with $N$ 


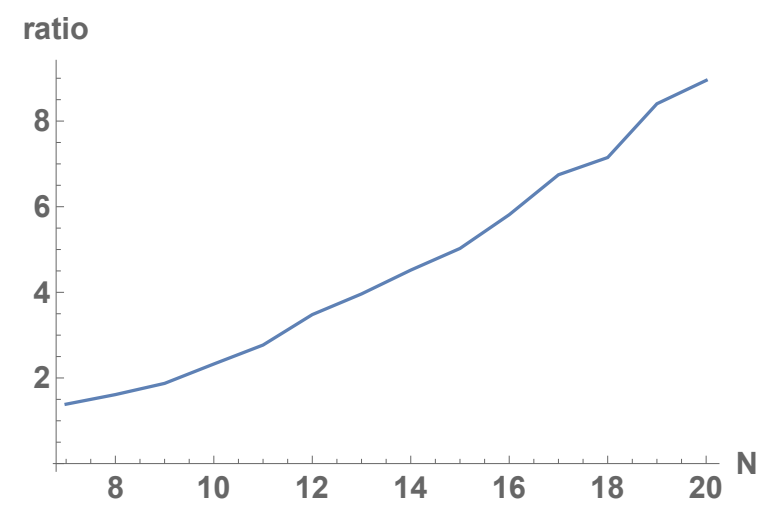

FIG. 18: Ratios of complexities between standard MC and multilevel MC (see (37)), for $N>l_{0}=6$. This figure corresponds to Example 6.

(the maximum in $x$ of (39) has been taken). We fix $N_{\infty}=100$ and consider $N \in[1,20]$. The estimates are computed with standard MC simulation, using 20,000 realizations. Exponential decrease of the bias error is perceived, since it presents a concave and decreasing shape in log-scale, disregarding the oscillations due to the finite number of realizations. Further, the exponential decay of the bias error was rigorously proved in [25, Remark 3.8].

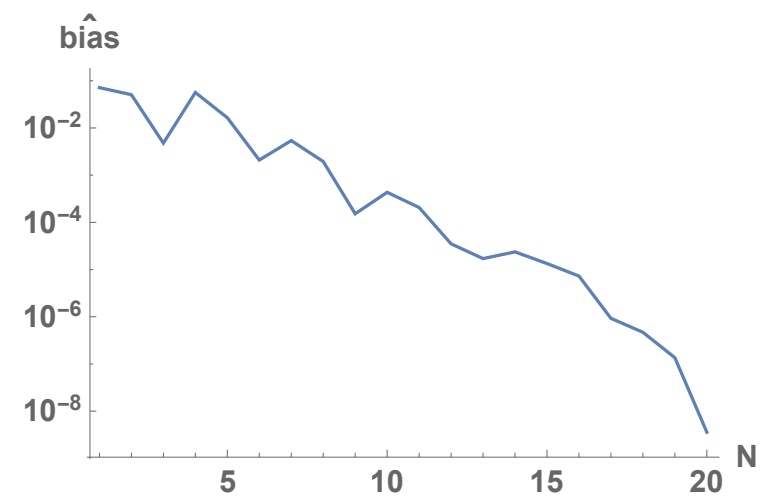

FIG. 19: Estimated bias errors with $N$ in log-scale. This figure corresponds to Example 6.

5

Suppose that we wish to approximate $f_{X(t=1.5)}(x)$ uniformly with theoretical root mean square error $\delta>0$. Let $\epsilon=\epsilon_{\text {bias }}=\epsilon_{\text {sampling }}=\delta / 2$. We pick the first $N=N(\epsilon)$ for which the estimated bias error is less than $\epsilon$. Multilevel $\mathrm{MC}$ can be applied with $l_{0}=6$, that $N(\epsilon)$, and sampling error $\epsilon_{\text {sampling }}^{2}=\epsilon^{2}$. In Figure 20, we illustrate the relation between $\epsilon$ and the complexities of multilevel and standard MC, with maximum level $N=N(\epsilon)$. The following approximate relations hold:

$$
W_{N(\epsilon)}^{\mathrm{MC}} \propto \epsilon^{-2} N(\epsilon)^{2}, \quad W_{l_{0}, N(\epsilon)}^{\text {multi }} \propto \epsilon^{-2} l_{0}^{2}
$$


(the symbol $\propto$ denotes proportionality to a constant). The cost of multilevel MC is reduced by factor $l_{0}^{2} / N(\epsilon)^{2}$. In $\log -\log$ scale, the complexity of multilevel MC increases linearly with respect to $\epsilon^{-1}$, with slope 2 . On the contrary, for standard MC, such relation is shifted up with $2 \log N(\epsilon)$. The sequence $N(\epsilon)$ increases very slowly with $\epsilon \rightarrow 0$, because of the fast decrease of the bias error towards 0 . But as $\epsilon \rightarrow 0$, multilevel MC becomes more and more efficient compared with standard MC.
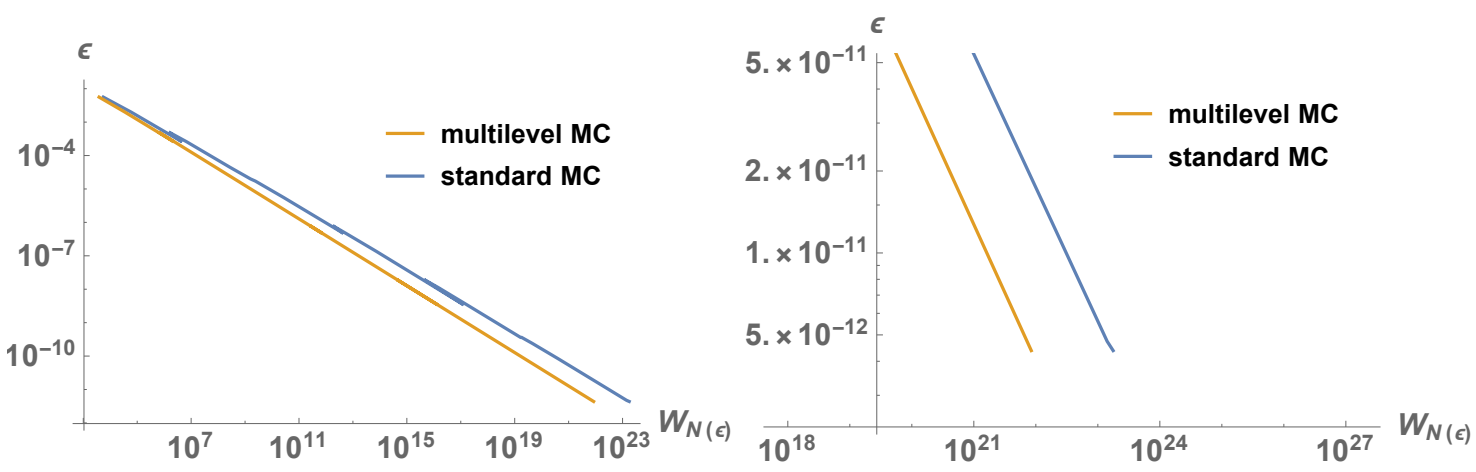

FIG. 20: Sampling error versus complexity (see (35)-(36)), in log-log scale. The right plot is a zoom. This figure corresponds to Example 6.

Let us approximate $f_{X(t=1.5)}(x)$ uniformly with theoretical root mean square error $\delta=0.001$. Let $\epsilon=\epsilon_{\text {bias }}=$ $\epsilon_{\text {sampling }}=\delta / 2=0.0005$. We pick the first $N$ for which the estimated bias error is less than $\epsilon: N=N(\epsilon)=9$. Then we apply the multilevel MC procedure with sampling error $\epsilon^{2}$. The optimal number of simulations for $N=9$ is the following: $M=\left(N_{6} ; M_{6}, M_{7}, M_{8}\right)=(19507 ; 1787,861,395)$. As expected, most of the cost is localized at level 6, while for the increments the cost goes down. The complexity of multilevel MC is $6.1 \times 10^{5}$, which is smaller than that of standard MC, $1.2 \times 10^{6}$. In Figure 21, left panel, we plot the estimate $\hat{f}_{X^{N=9}(t=1.5)}(x)$. It satisfies $\left\|f_{X(1.5)}(x)-\hat{f}_{X^{9}(1.5)}(x)\right\|_{2} \leq \delta$, for all $x \in \mathbb{R}$. In the right plot, we show the estimated densities for $N=8,9$ and 10 , with root mean square norm of the sampling error $\epsilon=0.0005$. Observe that the densities overlap, thus indicating the expected pointwise convergence.

Example 7. We study problem (16) with one degree polynomial coefficients. The probability distributions for the random inputs are $A_{0}=4, A_{1} \sim \operatorname{Uniform}(-3,1),\left.B_{0} \sim \operatorname{Gamma}(2,2)\right|_{[0,4]}, B_{1} \sim \operatorname{Bernoulli}(0.35)$ and $Y_{0}, Y_{1} \sim$ $\operatorname{Normal}(2, \sigma=3)$, all them independent. Due to the boundedness of $A_{0}, A_{1}, B_{0}$ and $B_{1}$, this problem has a unique analytic stochastic solution $X(t), t \in \mathbb{R}$, in the sense of the $\mathrm{L}^{p}(\Omega)$ random calculus, for $1 \leq p<\infty$.

The objective is to approximate the probability density function $f_{X(t)}(x)$ using the multilevel MC approach, by estimating $f_{X^{N}(t)}(x)$. The theoretical results guarantee that $\left\{f_{X^{N}(t)}(x)\right\}_{N \geq 0}$ converges to $f_{X(t)}(x)$ pointwise and in 


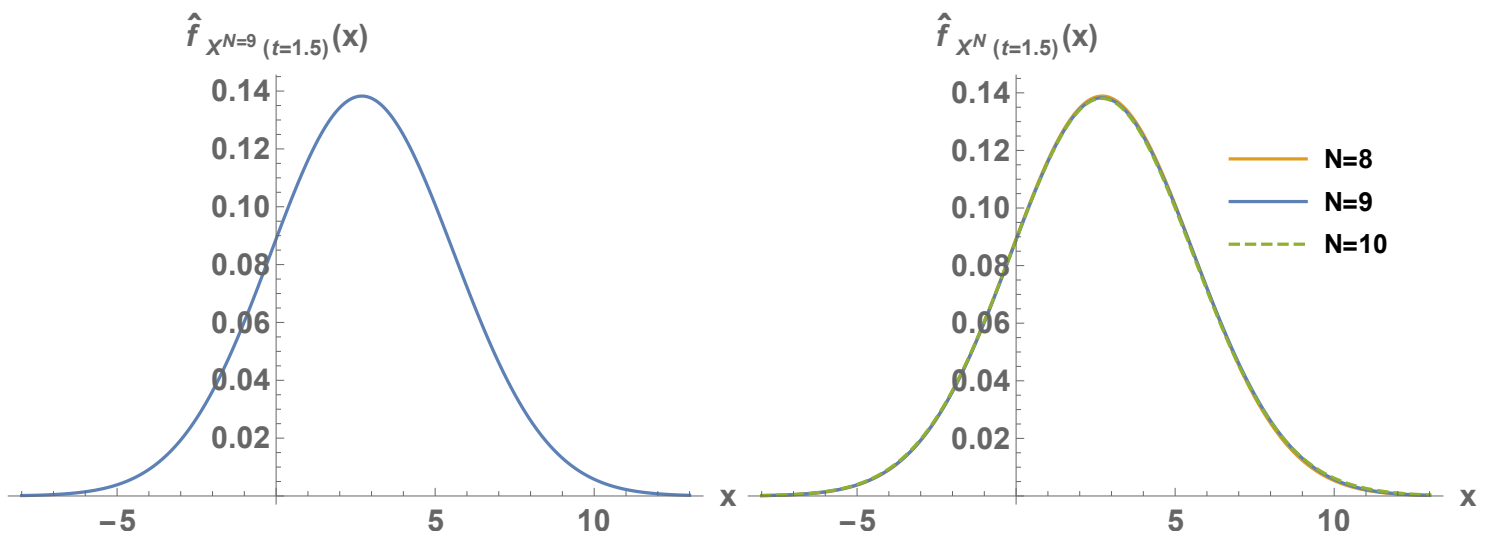

FIG. 21: Left plot: estimate $\hat{f}_{X^{9}(1.5)}(x)$. Right plot: estimates $\hat{f}_{X^{N}(1.5)}(x)$, for $N=8,9,10$. This figure corresponds to Example 6.

$\mathrm{L}^{p}(\mathbb{R}), 1 \leq p<\infty$, as $N \rightarrow \infty$. We work at time $t=1.5$.

We estimate $\mathbb{V}\left[Z_{\Delta}^{l}(x, t)\right]$ for $N$ large (till $N=70$ ), using parallel computing and $m_{\mathbb{V}}=50,000$ realizations. The number of realizations $m_{\mathbb{V}}$ is actually excessively large, but we want to be quite exact in this example to show accurate complexities.

With costs $c_{l}=C_{l}=l$, we obtain the ratios of complexities between standard MC and multilevel MC, see (37). In Figure 22, we vary the starting level $l_{1} \in[1,69]$ and the maximum level $N \in\left[l_{1}+1,70\right]$ and plot the corresponding ratios. Observe that the maximums (38) are greater than 1 (indicating that multilevel MC has higher efficiency) for $N \geq 36$ only, in contrast to Example 6 . This means that for $N<36$ and not sufficiently low tolerances crude MC simulation is more efficient. For each $N$, we keep $l_{0}$ maximizing the ratio, see (38). For $N=36$, the maximizer is $l_{0}=35$; for $N=37$ and 38 , the maximizer is $l_{0}=36$. For all $N \geq 39$, it is obtained the same $l_{0}=38$ as the maximizer.

In Figure 23 we report the ratios for $N>l_{0}=38$. The ratios are greater than 1 and, as $N$ increases, they tend to infinity at linear rate approximately, although with small slope $\approx 1 / l_{0}$. The complexity of standard MC grows linearly to infinity with $N$, while the complexity of multilevel MC becomes constant from a certain $N$. The oscillating behavior of the depicted ratios is due to the sampling error driven by the previous MC estimates for the variances. When $m_{\mathbb{V}}$ gets larger, the oscillations are mitigated and more exact results are obtained.

Let us approximate $f_{X(t=1.5)}(x)$ uniformly with root mean square error $\delta=0.0001$. Figure 24 shows the estimated bias errors (39), with $N_{\infty}=200$ and 20,000 MC simulations (the maximum in $x$ of (39) has been taken). We denote the bias errors as $\epsilon_{\text {bias }}^{N}$ (we make the dependence on $N$ explicit). The bias error needs quite large truncation order $N$ to start decreasing (up to $N=25$ the bias is 0.1 approximately), and for $N \geq 35$ it drops abruptly. The 


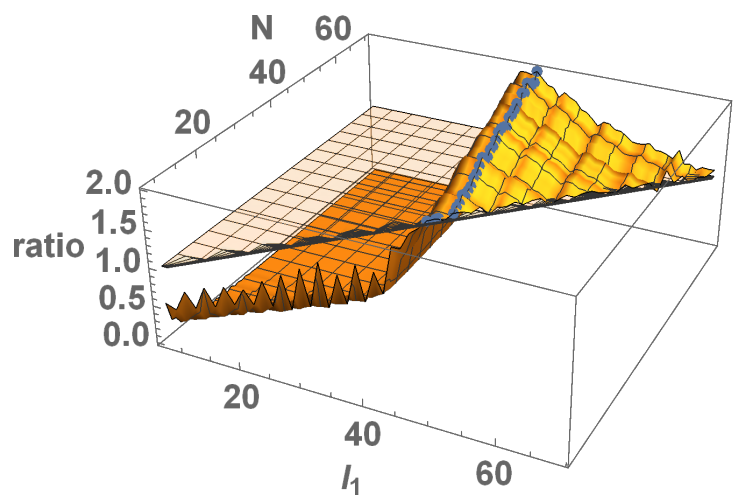

FIG. 22: Ratios of complexities between standard MC and multilevel MC (see (37)). The points indicate the maximum ratio with $l_{1}$ per truncation $N$. The transparent horizontal surface represents the ratio 1. This figure corresponds to Example 7.

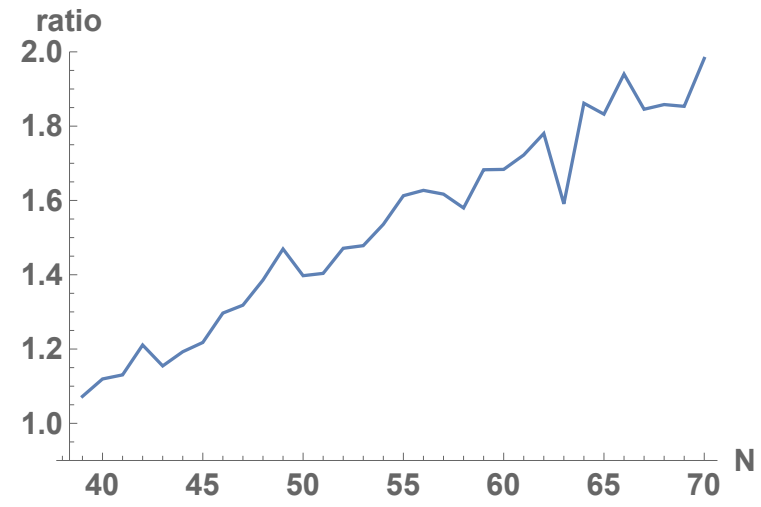

FIG. 23: Ratios of complexities between standard $\mathrm{MC}$ and multilevel $\mathrm{MC}$ (see (37)), for $N>l_{0}=38$. This figure corresponds to Example 7. 
bias error is less than $\delta$ only for $N \geq 37$, therefore we are in the region where multilevel MC is more efficient than standard MC. Notice that, in this example, we have fixed $\delta$ small on purpose to take advantage of the multilevel MC strategy. In the previous Example 6, we did not worry about $\delta$ nor its decomposition because multilevel MC showed more efficient than standard MC from the very first truncation order.

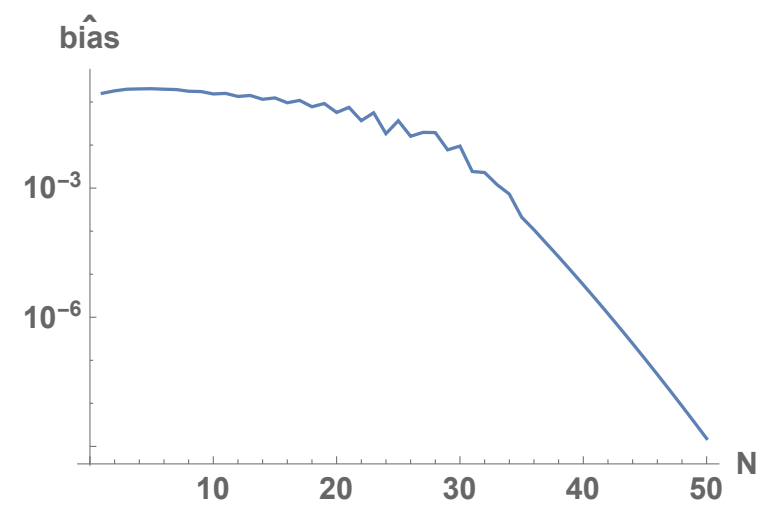

FIG. 24: Estimated bias errors with $N$ in log-scale. This figure corresponds to Example 7.

In Figure 25 we plot, with $N \geq 37$, the complexities of the multilevel and the standard MC approaches with $\epsilon_{\text {sampling }}=\delta-\epsilon_{\text {bias }}^{N}(\delta=0.0001)$, using (35)-(36). We observe that the complexity of multilevel MC is smaller, as expected. With formulas, we have

$$
W_{N}^{\mathrm{MC}}\left(\epsilon_{\text {sampling }}\right) \propto\left(\delta-\epsilon_{\text {bias }}^{N}\right)^{-2} N, \quad W_{l_{0}, N}^{\text {multi }}\left(\epsilon_{\text {sampling }}\right) \propto\left(\delta-\epsilon_{\text {bias }}^{N}\right)^{-2} l_{0}
$$

When $N \approx l_{0}$, we have $\delta-\epsilon_{\text {bias }}^{N} \approx 0$, so the complexities are large. When $N$ is large, then $\delta-\epsilon_{\text {bias }} \approx \delta$, so $W_{N}^{\mathrm{MC}}\left(\epsilon_{\text {sampling }}\right) \propto N \nearrow \infty$ and $W_{l_{0}, N}^{\text {multi }}\left(\epsilon_{\text {sampling }}\right) \searrow$ constant.

Given $\delta=0.0001$, in the case of standard MC the minimum complexity is attained when $N=43$ (bias $\epsilon_{\text {bias }}^{N=43}=$ $\left.5 \times 10^{-7}\right)$, this being $2 \times 10^{8}$.

For $N=50>l_{0}=38$, we run the multilevel MC procedure with sampling error $\epsilon_{\text {sampling }}^{2}=\left(\delta-\epsilon_{\text {bias }}^{N=50}\right)^{2}$. We choose $N=50$ because the corresponding cost of multilevel MC is uniformly cheaper than standard MC applied with any order of truncation. Also, the complexity of multilevel MC becomes constant for $N \geq 50$. For $N=50$, the optimal number of simulations is $M=\left(N_{38} ; M_{38}, \ldots, M_{50}\right)=(4527935 ; 12447,4977,2624,1507,475,292,126$, $45,22,9,4,2$ ), with a complexity that is $1.7 \times 10^{8}$. Figure 26 , left panel, plots the graph of the estimated density function $\hat{f}_{X^{N=50}(t=1.5)}(x)$, which approximates the true density $f_{X(t=1.5)}(x)$ with root mean square error less than $\delta$, for all $x \in \mathbb{R}$. The right plot depicts the density estimates for $N=49,50$ and 51, showing the expected overlapping. 


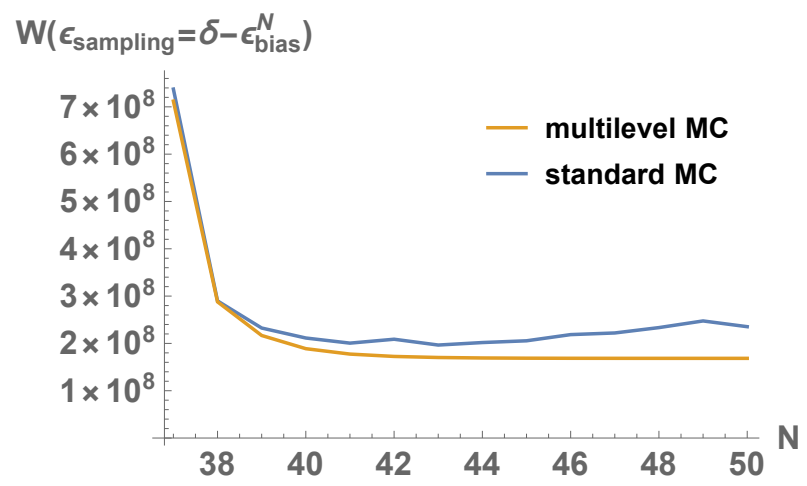

FIG. 25: Complexities of the multilevel and the standard MC approaches with $\epsilon_{\text {sampling }}=\delta-\epsilon_{\text {bias }}^{N}, \delta=0.0001$ fixed, using (35)-(36). This figure corresponds to Example 7.
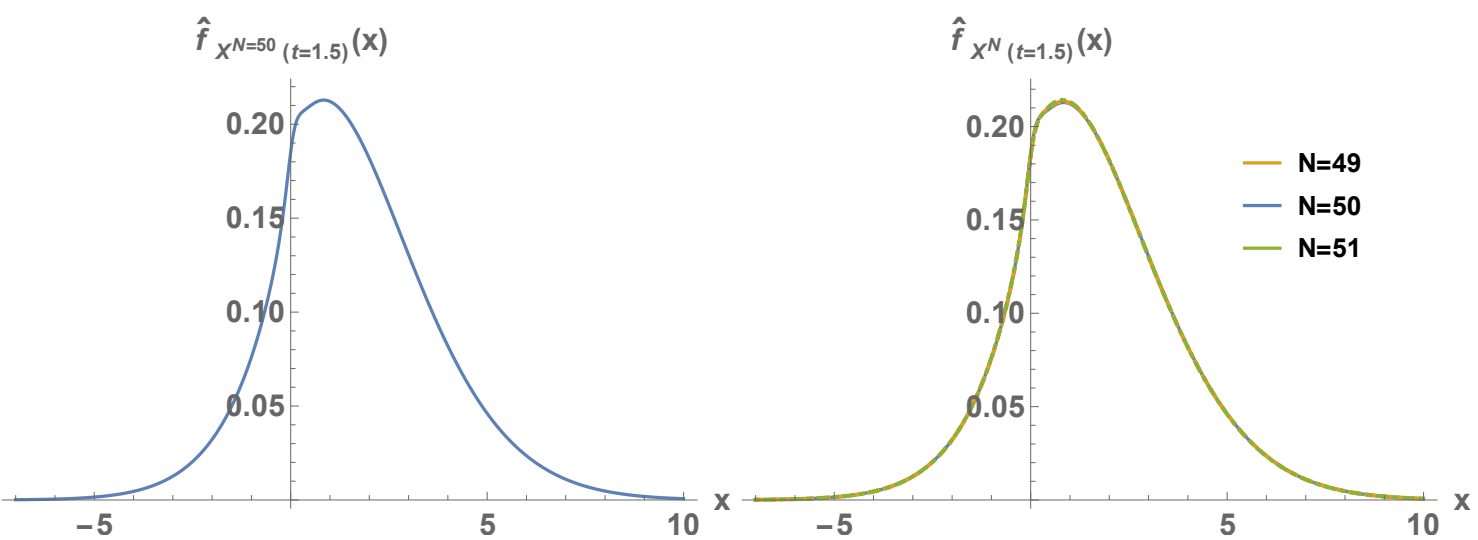

FIG. 26: Left plot: estimate $\hat{f}_{X^{50}(1.5)}(x)$. Right plot: estimates $\hat{f}_{X^{N}(1.5)}(x)$, for $N=49,50,51$. This figure corresponds to Example 7. 
Remark 2. Our application of the multilevel MC strategy is based on $\mathrm{L}^{\infty}(\mathbb{R})$ norms. First, we acted pointwise in $x$ in the constrained minimization problem from Proposition 5, and we took the $\mathrm{L}^{\infty}(\mathbb{R})$ norm of the optimal number of simulations. Then the target density function was approximated uniformly in $x$. Second, the ratio (37) of complexities considered the $\mathrm{L}^{\infty}(\mathbb{R})$ norms of the standard and the multilevel MC approaches.

Other norms can be considered. For instance, we could minimize the cost (31) given a fixed $\mathrm{L}^{1}(\mathbb{R})$ norm of the sampling error variance (30): $\int_{\mathbb{R}}\left\|f_{X^{N}(t)}(x)-f_{X}^{N, M}(x, t)\right\|_{2}^{2} \mathrm{~d} x=\epsilon_{\text {sampling }}^{2}$ In this case, we substitute each variance in $x$ by its integral on $\mathbb{R}$. Then the approach becomes independent of $x$ from the beginning.

When the cost is minimized pointwise in $x$, another possibility consists in considering the ratio of the $\mathrm{L}^{1}(\mathbb{R})$ norms of the complexities:

$$
W_{N}^{M C,\|\cdot\|_{1}}(t)=\epsilon_{\text {sampling }}^{-2} c_{N} \int_{\mathbb{R}} \mathbb{V}\left[Z^{N}(x, t)\right] \mathrm{d} x
$$

$$
W_{l_{1}, N}^{\text {multi, }\|\cdot\|_{1}}(t)=\epsilon_{\text {sampling }}^{-2} \int_{\mathbb{R}}\left(\sqrt{\mathbb{V}\left[Z^{l_{1}}(x, t)\right] c_{l_{1}}}+\sum_{l=l_{1}}^{N-1} \sqrt{\mathbb{V}\left[Z_{\Delta}^{l}(x, t)\right] C_{l}}\right)^{2} \mathrm{~d} x,
$$

$$
R_{l_{1}, N}^{\|\cdot\|_{1}}(t)=\frac{W_{N}^{M C,\|\cdot\|_{1}}(t)}{W_{l_{1}, N}^{m u l t i,\|\cdot\|_{1}}(t)}
$$

(the ratio is independent of $\epsilon_{\text {sampling }}$ ). In the two examples from this section, the conclusions derived are analogous considering $\mathrm{L}^{1}(\mathbb{R})$ norms, thus showing robustness.

We present results regarding Example 6. Using $m_{\mathbb{V}}=10,000$ realizations and costs $c_{l}=C_{l}=l^{2}$, we estimate the ratios of complexities (43) for $l_{1} \in[1,19]$ and $N \in\left[l_{1}+1,20\right]$. Figure 27, left panel, shows the ratios. These ratios show a similar pattern to Figure 17 . For each $N$, we keep the level $l_{0}$ maximizing the ratio (43). For $N \geq 7$, the same maximizer $l_{0}=6$ is obtained, as in Example 6. The right panel of Figure 27 compares the two ratios (37) and (43) for $N \geq l_{0}=6$. The two ratios are very similar, only showing a small discrepancy in part due to the MC procedure with $m_{\mathbb{V}}$ realizations. The fact that the two ratios are practically coinciding is justified theoretically by the approximation $R_{l_{1}, N}^{\|\cdot\|_{1}}(t) \approx N^{2} / l_{1}^{2}$, which also held for (37).

Figure 28, left panel, analyzes theoretical error against complexity (see (41)-(42)). It corresponds to Figure 20 by considering $\mathrm{L}^{1}(\mathbb{R})$ norms, instead. When changing the norm, the "line" corresponding to error versus complexity in log-log scale is shifted up or down but has the same slope. This is because changing the norm only modifies the proportionality constants in (40). This is illustrated in Figure 28, right panel, where the two "lines" corresponding to multilevel MC are parallel, and also for standard MC. 


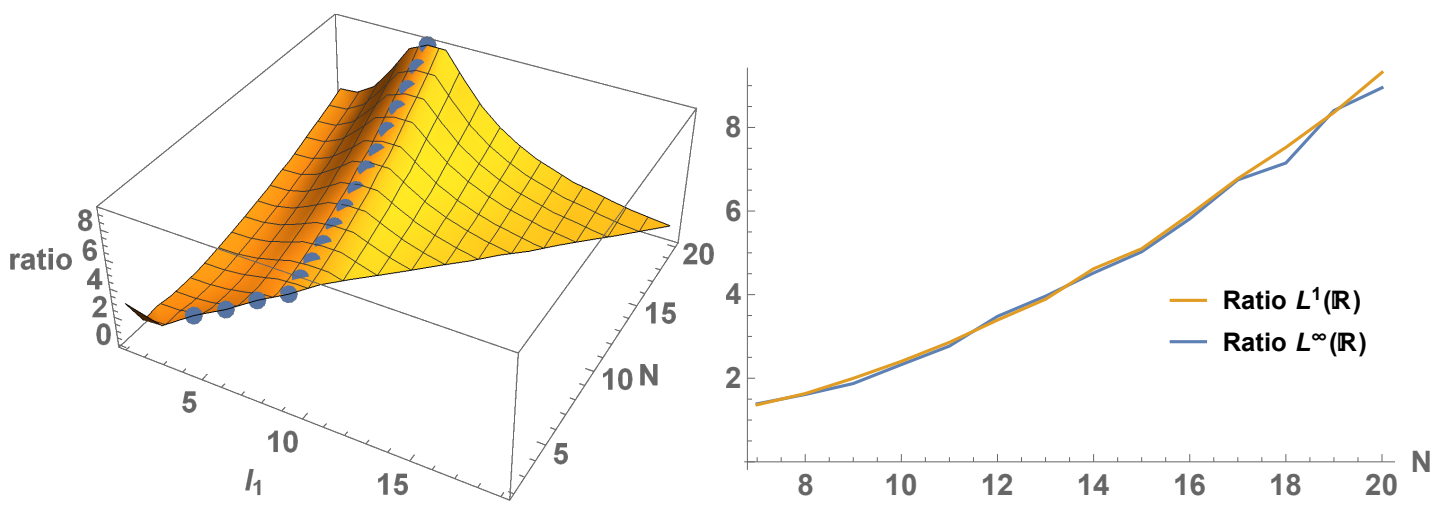

FIG. 27: Left panel: ratios of the $\mathrm{L}^{1}(\mathbb{R})$ norms of the complexities between standard and multilevel MC (see (43)), where the points indicate the maximum ratio with $l_{1}$ per truncation $N$. Right panel: ratios of complexities between standard and multilevel MC with $\mathrm{L}^{\infty}(\mathbb{R})$ norms (see (37)) and $\mathrm{L}^{1}(\mathbb{R})$ norms (see (43)), for $N>l_{0}=6$. This figure corresponds to Remark 2.
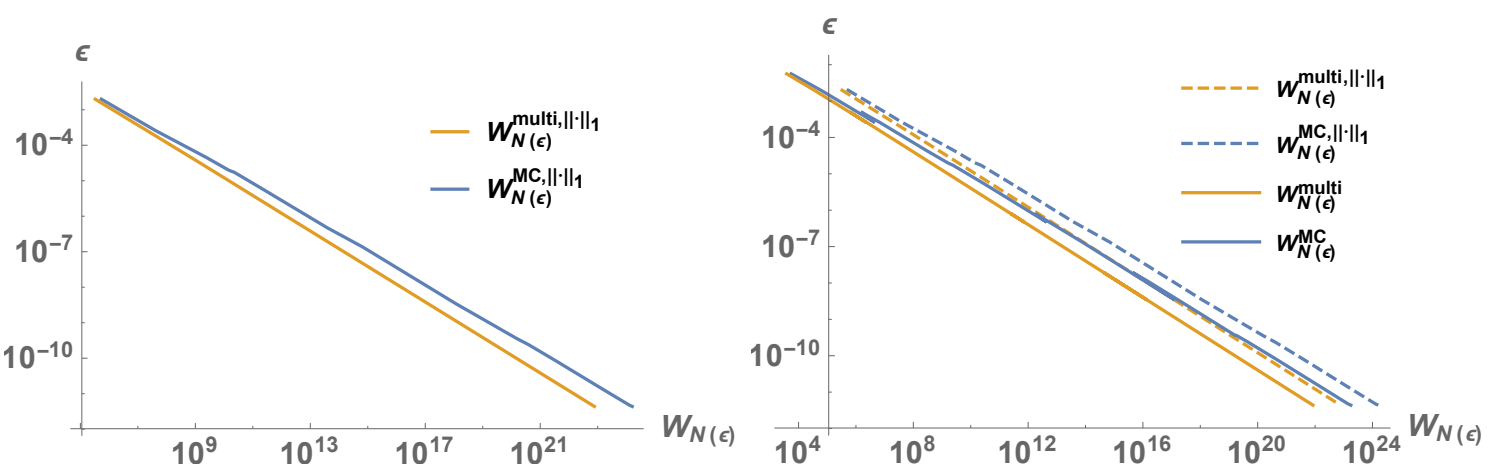

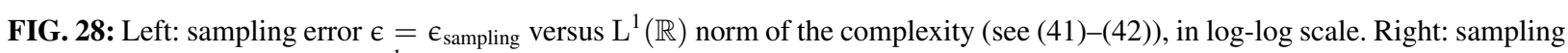
error $\epsilon=\epsilon_{\text {sampling }}$ versus the $L^{1}(\mathbb{R})$ (see (41)-(42)) and $L^{\infty}(\mathbb{R})$ (see (35)-(36)) norms of the complexity, in log-log scale. This figure corresponds to Remark 2 . 


\section{CONCLUSIONS}

This work focused on the improvement of the computational methods and algorithms from the recent contribution [25]. In [25], the authors studied the random second order linear differential equation with analytic data processes and random initial conditions, and constructed approximations to the probability density function of the stochastic solution based on dimensionality reduction and standard MC sampling strategy. The main goal in this paper is to speed up the convergence of the standard MC algorithm used in [25] by controlling the variance of the statistical estimators, thus avoiding noisy features in the density estimates.

The path-wise selection of the initial condition used in the density expression leads to a stable algorithm with good convergence. It was proved theoretically that the statistical estimators constructed with this method always possess finite variance. The implementation of the algorithm was illustrated through simple autonomous and nonautonomous linear differential equations.

Improvements are achieved by computing the expectation with respect to the initial conditions via numerical integration methods. By considering the densities of the initial conditions as integration weights, we focused on Gaussian quadrature rules. The orthogonal polynomials were considered with respect to standard probability distributions. Several conclusions are reached from this approach. When the initial conditions are comparable with the input coefficients of the equation in terms of random variability, the quadrature rule for integration allows significant variance reduction of the statistical estimators. Even when the initial conditions have small dispersion compared to the input coefficients, if the cost of each realization of the fundamental set is quite expensive, the quadrature rule yields similar expense to a plain MC strategy. The quadrature degree has to be selected sufficiently large to assure error-free approximations of the integrals.

A multilevel version of the $\mathrm{MC}$ algorithm allows for reduction of the complexity, by linear or quadratic factor. The multilevel MC approach turns out to be useful when high accuracy in the density approximation is required. The starting level was selected on the fly to maximize the ratio of complexities between the standard and the multilevel MC approach. Once the initial level is selected, and given a sampling error variance, we obtained the optimal number of simulations per level to minimize the complexity. The numerical results illustrated the methodology step-by-step in order to show the improvement with regard to the standard version of the MC simulation algorithm.

Several fundamental extensions and algorithmic improvements may be conceived, especially concerning the multilevel MC methodology. Firstly, the three parts in which we divided the multilevel procedure (variance of the increments, selection of the initial level by maximizing the ratio, and approximation of the density) could be combined in an automated algorithm, which would obtain the bias error, the sampling error, the variances, the initial level and the 
optimal number of simulations on the fly. Secondly, the multilevel MC approach may be combined with a numerical integration scheme for the expectation with respect to the initial conditions. Finally, our multilevel strategy relied on considering consecutive levels, while other strategies could utilize different sequences of levels, such as linear or geometric ones.

An interesting area to explore is the extension of the presently methodology to linear systems of second order random differential equations. In this case, the aim is to approximate the joint probability density function of the vector-valued stochastic solution. With the exception of simple autonomous linear systems whose density function can be computed through transformations of random variables/vectors and direct integration, no analysis has been carried out for non-autonomous systems. The application to non-autonomous systems would offer promising advances in the active field of random differential equations.

\section{ACKNOWLEDGEMENTS}

This work is supported by Spanish "Ministerio de Economía y Competitividad” grant MTM2017-89664-P. M. Jornet acknowledges the doctorate scholarship granted by PAID, as well as "Ayudas movilidad de estudiantes de doctorado de la Universitat Politècnica de València para estancias en 2019”, for financing his research stay at CMAP. J. Calatayud acknowledges "Fundació Ferran Sunyer i Balaguer", "Institut d'Estudis Catalans" and the award from "Borses Ferran Sunyer i Balaguer 2019" for funding her research stay at CMAP. All authors are grateful to Inria (Centre de Saclay, DeFi Team), which hosted M. Jornet and J. Calatayud during their research stays at École Polytechnique. The authors thank the reviewers for the valuable comments and suggestions, which have greatly enriched the quality of the paper.

\section{CONFLICT OF INTEREST STATEMENT}

The authors declare that there is no conflict of interests regarding the publication of this article.

\section{APPENDIX: PROOFS}

\section{Proofs for Section 2.}

Lemma 6. Let $U$ be an absolutely continuous random variable, $\left(Z_{1}, Z_{2}\right)$ be a random vector such that $Z_{1} \neq 0$ almost surely, and $G \in \mathcal{F}$ be an event of non-zero probability. Suppose that $U$ and $\left(Z_{1}, Z_{2}, 1_{G}\right)$ are independent. Then the 
1 probability law of $Z_{1} U+Z_{2}$ conditioned to $G$ is absolutely continuous, with density function

$$
f_{Z_{1} U+Z_{2}}(z \mid G)=\mathbb{E}\left[f_{U}\left(\frac{z-Z_{2}}{Z_{1}}\right) \frac{1}{\left|Z_{1}\right|} \mid G\right]
$$

Proof. On the measurable space $(\Omega, \mathcal{F})$, define the conditional probability measure $\mathbb{P}_{G}$ as $\mathbb{P}_{G}[E]=\mathbb{P}[E \mid G]:=$ $\mathbb{P}[E \cap G] / \mathbb{P}[G]$, for $E \in \mathcal{F}$.

Since $U$ and $G$ are independent, the probability law of $U$ with respect to $\mathbb{P}_{G}$ coincides with $\mathbb{P}$. Then the law of $U$ under $\mathbb{P}_{G}$ is absolutely continuous, and $f_{U}(u \mid G)=f_{U}(u)$.

On the other hand, notice that $U$ and $\left(Z_{1}, Z_{2}\right)$ are independent under $\mathbb{P}_{G}$.

Finally, $\mathbb{P}_{G}$ is absolutely continuous with respect to $\mathbb{P}, \mathbb{P}_{G} \ll \mathbb{P}$, therefore $Z_{1} \neq 0 \mathbb{P}_{G}$-almost surely.

Thus, on the probability space $\left(\Omega, \mathcal{F}, \mathbb{P}_{G}\right)$ we are in conditions of applying [25, Lemma 3.2]:

$$
f_{Z_{1} U+Z_{2}}(z \mid G)=\mathbb{E}\left[f_{U}\left(\frac{z-Z_{2}}{Z_{1}} \mid G\right) \frac{1}{\left|Z_{1}\right|} \mid G\right]=\mathbb{E}\left[f_{U}\left(\frac{z-Z_{2}}{Z_{1}}\right) \frac{1}{\left|Z_{1}\right|} \mid G\right]
$$

Proof of Proposition 1. We focus on the equality $f_{X(t)}(x)=\mathbb{E}[Z(x, t)]$, as the other one concerning $f_{X^{N}(t)}(x)$ is analogous.

If either $\mathbb{P}\left[G_{0}(t)\right]=0$ or $\mathbb{P}\left[G_{1}(t)\right]=0$, then $Z(x, t)=Z_{1}(x, t)$ almost surely or $Z(x, t)=Z_{0}(x, t)$ almost surely, respectively, so we are done.

Let us then assume that both $G_{0}(t)$ and $G_{1}(t)$ have non-zero probability. By the law of total probability, $f_{X(t)}(x)=$ $f_{X(t)}\left(x \mid G_{0}(t)\right) \mathbb{P}\left[G_{0}(t)\right]+f_{X(t)}\left(x \mid G_{1}(t)\right) \mathbb{P}\left[G_{1}(t)\right]$. To compute the conditional densities, we use Lemma 6:

$$
f_{X(t)}\left(x \mid G_{0}(t)\right)=\mathbb{E}\left[Z_{0}(x, t) \mid G_{0}(t)\right], f_{X(t)}\left(x \mid G_{1}(t)\right)=\mathbb{E}\left[Z_{1}(x, t) \mid G_{1}(t)\right]
$$

As a consequence, $f_{X(t)}(x)=\mathbb{E}[Z(x, t)]$.

Proof of Lemma 2. Fix $T>t_{0}$ and $t \in\left[t_{0}, T\right]$. Consider the Wronskian $W_{S_{0}, S_{1}}(t)=S_{0}(t) \dot{S}_{1}(t)-S_{1}(t) \dot{S}_{0}(t)$, where the derivatives are regarded in the $\mathrm{L}^{\infty}(\Omega)$ sense. Recall that $S_{0}(t)$ and $S_{1}(t)$ solve the differential equation in the $\mathrm{L}^{\infty}(\Omega)$ sense, see Section 1. In particular, the sample paths of $S_{0}(t)$ and $S_{1}(t)$ solve the deterministic version of the differential equation, for almost every $\omega \in \Omega$. By Liouville's formula for deterministic linear differential equations [38, Prop. 2.15], $W_{S_{0}, S_{1}}(t)=\mathrm{e}^{-\int_{t_{0}}^{t} A(s) \mathrm{d} s}$ almost surely. As $A(t)$ is a power series in $\mathrm{L}^{\infty}(\Omega)$, we can lower bound 
1 the Wronskian as follows:

$$
W_{S_{0}, S_{1}}(t) \geq \mathrm{e}^{-\int_{t_{0}}^{T}\|A(s)\|_{\infty} \mathrm{d} s}=: \beta>0
$$

almost surely. On the other hand, using the triangular inequality,

$$
\begin{aligned}
W_{S_{0}, S_{1}}(t) & \leq 2 \max \left\{\left|S_{0}(t)\right|,\left|S_{1}(t)\right|\right\} \max \left\{\left|\dot{S}_{0}(t)\right|,\left|\dot{S}_{1}(t)\right|\right\} \\
& \leq 2 \max \left\{\left|S_{0}(t)\right|,\left|S_{1}(t)\right|\right\} \max \left\{\left\|\dot{S}_{0}(t)\right\|_{\infty},\left\|\dot{S}_{1}(t)\right\|_{\infty}\right\} \\
& \leq 2 \max \left\{\left|S_{0}(t)\right|,\left|S_{1}(t)\right|\right\} \gamma,
\end{aligned}
$$

2 where $\gamma$ is a constant upper bound for $\left\|\dot{S}_{0}(t)\right\|_{\infty}$ and $\left\|\dot{S}_{1}(t)\right\|_{\infty}$ on $\left[t_{0}, T\right]$. As a consequence,

$$
\max \left\{\left|S_{0}(t)\right|,\left|S_{1}(t)\right|\right\} \geq \frac{\beta}{2 \gamma}=: 2 \alpha
$$

Proof of Proposition 3. Observe that, by (11) and (12),

$$
\left|Z^{N}(x, t)\right| \leq \max \left\{\left\|f_{Y_{0}}\right\|_{\infty},\left\|f_{Y_{1}}\right\|_{\infty}\right\}\left(\frac{1}{\left|S_{0}^{N}(t)\right|} 1_{G_{0}^{N}(t)}+\frac{1}{\left|S_{1}^{N}(t)\right|} 1_{G_{1}^{N}(t)}\right)=\frac{\max \left\{\left\|f_{Y_{0}}\right\|_{\infty},\left\|f_{Y_{1}}\right\|_{\infty}\right\}}{\max \left\{\left|S_{0}^{N}(t)\right|,\left|S_{1}^{N}(t)\right|\right\}}
$$

6 for $N \leq \infty$. Then Lemma 2 applies.

\section{Proofs for Section 4.}

Proof of Proposition 4. First, by Proposition $1, \mathbb{E}\left[Z^{l_{0}}(x, t)\right]=f_{X^{l_{0}}(t)}(x)$. On the other hand,

$$
\begin{aligned}
\mathbb{E}\left[Z_{\Delta}^{l}(x, t)\right] & =\mathbb{E}\left[\Delta Z_{0}^{l}(x, t) 1_{H_{0}^{l}(t)}\right]+\mathbb{E}\left[\Delta Z_{1}^{l}(x, t) 1_{H_{1}^{l}(t)}\right] \\
& =\mathbb{E}\left[\Delta Z_{0}^{l}(x, t) \mid H_{0}^{l}(t)\right] \mathbb{P}\left[H_{0}^{l}(t)\right]+\mathbb{E}\left[\Delta Z_{1}^{l}(x, t) \mid H_{1}^{l}(t)\right] \mathbb{P}\left[H_{1}^{l}(t)\right] \\
& =\mathbb{E}\left[Z_{0}^{l+1}(x, t) \mid H_{0}^{l}(t)\right] \mathbb{P}\left[H_{0}^{l}(t)\right]-\mathbb{E}\left[Z_{0}^{l}(x, t) \mid H_{0}^{l}(t)\right] \mathbb{P}\left[H_{0}^{l}(t)\right] \\
& +\mathbb{E}\left[Z_{1}^{l+1}(x, t) \mid H_{1}^{l}(t)\right] \mathbb{P}\left[H_{1}^{l}(t)\right]-\mathbb{E}\left[Z_{1}^{l}(x, t) \mid H_{1}^{l}(t)\right] \mathbb{P}\left[H_{1}^{l}(t)\right] .
\end{aligned}
$$


By Lemma 6 and the law of total probability,

$$
\begin{aligned}
\mathbb{E}\left[Z_{\Delta}^{l}(x, t)\right] & =f_{X^{l+1}(t)}\left(x \mid H_{0}^{l}(t)\right) \mathbb{P}\left[H_{0}^{l}(t)\right]-f_{X^{l}(t)}\left(x \mid H_{0}^{l}(t)\right) \mathbb{P}\left[H_{0}^{l}(t)\right] \\
& +f_{X^{l+1}(t)}\left(x \mid H_{1}^{l}(t)\right) \mathbb{P}\left[H_{1}^{l}(t)\right]-f_{X^{l}(t)}\left(x \mid H_{1}^{l}(t)\right) \mathbb{P}\left[H_{1}^{l}(t)\right] \\
& =f_{X^{l+1}(t)}(x)-f_{X^{l}(t)}(x) .
\end{aligned}
$$

1 The proposition follows from telescopic sum identities.

\section{REFERENCES}

1. Soong, T.T., Random Differential Equations in Science and Engineering, New York: Academic Press, 1973.

2. Neckel, T. and Rupp, F., Random Differential Equations in Scientific Computing, London: Walter de Gruyter, 2013.

3. Strand, J.L., Random ordinary differential equations, J. Differ. Equ., 7, pp. 538-553, 1970.

4. Smith, R.C., Uncertainty Quantification. Theory, Implementation, and Application, New York: SIAM Computational Science \& Engineering, 2014.

5. Cortés, J.-C., Jódar, L., Camacho, J. and Villafuerte, L., Random Airy type differential equations: Mean square exact and numerical solutions, Comput. Math. Appl., 60, pp. 1237-1244, 2010.

6. Calbo, G., Cortés, J.-C. and Jódar, L., Random Hermite differential equations: mean square power series solutions and statistical properties, Appl. Math. Comput., 218(7), pp. 3654-3666, 2011.

7. Calatayud, J., Cortés, J.-C. and Jornet, M., Improving the approximation of the first- and second-order statistics of the response stochastic process to the random Legendre differential equation, Mediterr. J. Math., 16(68), pp. 1-14, 2019.

8. Calatayud, J., Cortés, J.-C. and Jornet, M., Computational uncertainty quantification for random non-autonomous second order linear differential equations via adapted gPC: a comparative case study with random Fröbenius method and Monte Carlo simulation, Open Math., 16, pp. 1651-1666, 2018.

9. Xiu, D., Numerical Methods for Stochastic Computations. A Spectral Method Approach, New York: Cambridge Texts in Applied Mathematics, Princeton University Press, 2010.

10. Golmankhaneh, A.A., Porghoveh, N.A. and Baleanu, D., Mean square solutions of second-order random differential equations by using homotopy analysis method, Rom. Rep. Phys., 65, pp. 350-362, 2013.

11. Khudair, A.R., Ameen, A.A. and Khalaf, S.L., Mean square solutions of second-order random differential equations by using variational iteration method, Appl. Math. Sci., 5, pp. 2505-2519, 2011.

12. Khudair, A.R., Ameen, A.A. and Khalaf, S.L., Mean square solutions of second-order random differential equations by using 
Adomian decomposition method, Appl. Math. Sci., 5, pp. 2521-2535, 2011.

13. Khudair, A.K., Haddad, S.A.M. and Khalaf, S.L., Mean square solutions of second-order random differential equations by using the differential transformation method, Open Journal of Applied Sciences, 6, pp. 287-297, 2016.

14. Villafuerte, L. and Chen-Charpentier, B.M., A random differential transform method: Theory and applications, Appl. Math. Lett., 25, pp. 1490-1494, 2012.

15. Calatayud, J., Cortés, J.-C., Jornet, M. and Villafuerte, L., Random non-autonomous second order linear differential equations: mean square analytic solutions and their statistical properties, Adv. Differ. Equ., 2018(392), pp. 1-29, 2018.

16. Calatayud, J., Cortés, J.-C. and Jornet, M., Some notes to extend the study on random non-autonomous second order linear differential equations appearing in mathematical modeling, Mathematical and Computational Applications, 23(4), pp. 76-89, 2018.

17. Cortés, J.-C., Navarro-Quiles, A., Romero, J.-V. and Roselló, M.-D., Probabilistic solution of random autonomous first-order linear systems of ordinary differential equations, Rom. Rep. Phys., 68(4), pp. 1397-1406, 2016.

18. Calatayud, J., Chen-Charpentier, B.M., Cortés, J.-C. and Jornet, M., Combining polynomial chaos expansions and the random variable transformation technique to approximate the density function of stochastic problems, including some epidemiological models, Symmetry, 11(1), pp. 43-60, 2019.

19. El-Tawil, M.A., The approximate solutions of some stochastic differential equations using transformations, Appl. Math. Comput., 164(1), pp. 167-178, 2005.

20. Dorini, F.A., Cecconello, M.S. and Dorini, M.B., On the logistic equation subject to uncertainties in the environmental carrying capacity and initial population density, Commun. Nonlinear Sci. Numer. Simul. 33, pp. 160-173, 2016.

21. Santos, L.T., Dorini, F.A. and Cunha, M.C.C., The probability density function to the random linear transport equation, Appl. Math. Comput., 216(5), pp. 1524-1530, 2010.

22. Calatayud, J., Cortés, J.-C. and Jornet, M., The damped pendulum random differential equation: A comprehensive stochastic analysis via the computation of the probability density function, Physica A, 512, 261-279, 2018.

23. Hussein, A. and Selim, M.M., Solution of the stochastic radiative transfer equation with Rayleigh scattering using RVT technique, Appl. Math. Comput., 218(13), pp. 7193-7203, 2012.

24. Casabán, M.C., Cortés, J.-C., Romero, J.V. and Roselló, M.D., Solving random homogeneous linear second-order differential equations: a full probabilistic description, Mediterr. J. Math., 13(6), pp. 3817-3836, 2016.

25. Jornet, M., Calatayud, J., Le Maître, O.P. and Cortés, J.-C., Second order linear differential equations with analytic uncertainties: Stochastic analysis via the computation of the probability density function, J. Comput. Appl. Math., 374(112770), pp. $1-20,2020$.

26. Wolfram Research, Inc., Mathematica, Version 11.2, Champaign, IL, USA: Wolfram Research, Inc., 2017.

Volume x, Issue x, 2017 
1 27. Giles, M.B., Multilevel Monte Carlo path simulation, Oper. Res., 56(3), pp. 607-617, 2008.

2 28. Giles, M.B., Multilevel Monte Carlo methods, Acta Numer., 24, pp. 259-328, 2015.

3

29. Cliffe, A., Giles, M.B., Scheichl, R. and Teckentrup, A., Multilevel Monte Carlo Methods and applications to elliptic PDEs with random coefficients, Computing and Visualization in Science, 14(1), pp. 3-15, 2011.

30. Pisaroni, M., Nobile, F.B. and Leyland, P., A Continuation Multi Level Monte Carlo method for uncertainty quantification in compressible inviscid aerodynamics, Comput. Method. Appl. M., 326(C): pp. 20-50, 2017.

31. Beskos, A., Jasra, A., Law, K., Tempone, R. and Zhou, Y., Multilevel sequential Monte Carlo samplers, Stoch. Proc. Appl., 127(5), pp. 1417-1440, 2017.

32. Sobol, I.M., Global sensitivity indices for nonlinear mathematical models and their Monte Carlo estimates, Math. Comput. Simulat., 55(1-3), pp. 271-280, 2001.

33. Saltelli, A., Ratto, M., Andres, T., Campolongo, F., Cariboni, J., Gatelli, D., Saisana, M. and Tarantola, S., Global Sensitivity Analysis, The Primer, England: John Wiley \& Sons, 2008.

34. Saltelli, A., Annoni, P., Azzini, I., Campolongo, F., Ratto, M. and Tarantola, S., Variance based sensitivity analysis of model output. Design and estimator for the total sensitivity index, Comput. Phys. Commun., 181(2), pp. 259-270, 2010.

35. Sobol', I.M., Tarantola, S., Gatelli, D., Kucherenko, S. and Mauntz, W., Estimating the approximation error when fixing unessential factors in global sensitivity analysis, Reliab. Eng. Syst. Safe., 92(7), pp. 957-960, 2007.

36. Efron, B. and Tibshirani, R., An Introduction to the Bootstrap, New York: CRC Press, 1994.

37. Davison, A.C. and Hinkley D.V., Bootstrap Methods and Their Application, London: Cambridge University Press, 1997.

38. Chicone, C., Ordinary Differential Equations With Applications, New York: Springer Science+Business Media, 2006. 